\title{
Ascomicetos del bosque mesófilo de montaña de Honey, Puebla de los Ángeles, México
}

\author{
Ascomycetes from the tropical cloud forest of Honey, \\ Puebla de los Angeles, Mexico
}

\section{Acta Botanica Mexicana}

\author{
Marcos Sánchez Flores'1,2,3 (D), Ricardo Valenzuela' (D), Marco A. Hernández-Muñoz² (D), Jesús García Jiménez³ (iD) \\ Michelle Martínez-Pinedal (iD, Tania Raymundo',4 (iD
}

\section{Resumen:}

Antecedentes y Objetivos: Los ascomicetos son un grupo de hongos que se caracteriza por la formación de ascosporas dentro de ascas; se localizan en todos los ecosistemas terrestres y marinos. Los estudios de este grupo en el bosque mesófilo en México se han ido incrementando y en esta ocasión se presenta un inventario micoflorístico del municipio Honey, localizado en la Sierra Norte de Puebla, el cual conserva $50 \%$ de su vegetación original.

Métodos: Los hongos fueron recolectados en seis localidades del municipio Honey, durante los años 2016 al 2018 . Los especímenes fueron estudiados y determinados de acuerdo con las técnicas tradicionales en micología y se depositaron en las colecciones de hongos de los herbarios ENCB y FEZA.

Resultados clave: Se determinaron 52 especies de ascomicetos para el municipio Honey, de los cuales 39 son nuevos registros para el estado, mientras que Adelphella babingtonii, Cudoniella acicularis, Lachnum fuscescens, Ophioceras leptosporum, Orbilia curvatispora y Unguiculariopsis acerina lo son para México. Además, Hymenoscyphus herrerae se describe como especie nueva para la ciencia. De tal forma que con el presente estudio se tiene un total de 93 especies para la entidad y 141 para los bosques mesófilos del país. La familia Xylariaceae presentó la mayor riqueza taxonómica con 16 especies, siendo Xylaria con 14, el género mejor representado (27\%) de los ascomicetos del bosque mesófilo de Honey.

Conclusiones: Los ascomicetos son el grupo de hongos mejor estudiados en los bosques mesófilos de Puebla; no obstante, es necesario incrementar los esfuerzos para inventariar y describir la riqueza fúngica y de otros organismos de este ecosistema amenazado.

Palabras clave: Leotiomycetes, nuevos registros, Orbiliomycetes, Pezizomycetes, Sordariomycetes.

\section{Abstract:}

Background and Aims: Ascomycetes are a group of fungi that are characterized by the formation of ascospores within ascas, they occur in all terrestrial and marine ecosystems. The studies of this group in the cloud forest in Mexico have been increasing and a mycofloristic inventory of the municipality of Honey in the Sierra Norte in Puebla, which preserves $50 \%$ of its original vegetation, is presented here.

Methods: The specimens were collected in six locations in the municipality of Honey, during the years 2016 to 2018 . The specimens were studied and determined according to traditional techniques in mycology and were deposited in the fungus collections of the herbaria ENCB and FEZA.

Key results: Fifty-two species of ascomycetes were determined for the municipality of Honey, of which 39 are new records for the state, while Adelphella babingtonii, Cudoniella acicularis, Lachnum fuscescens, Ophioceras leptosporum, Orbilia curvatispora and Unguiculariopsis acerina are new records for Mexico. Additionally, Hymenoscyphus herrerae is described as a new species for science. Hence, with this study there are a total of 93 species for the entity and 141 for the cloud forest from Mexico. The family Xylariaceae presented the highest taxonomic richness with 16 species, Xylaria with 14 being the best represented genus (27\%) of ascomycetes from the cloud forest of Honey.

Conclusions: The Ascomycetes are the best studied group of fungi in the cloud forest from Puebla; however, it is necessary to increase efforts to inventory and describe the richness of fungi and other organisms in this endangered ecosystem.

Key words: Leotiomycetes, new records, Orbiliomycetes, Pezizomycetes, Sordariomycetes.

${ }^{1}$ Instituto Politécnico Nacional, Escuela Nacional de Ciencias Biológicas, Laboratorio de Micología, Prolongación de Carpio y Plan de Ayala, Santo Tomás, Alcaldía Miguel Hidalgo, 11340 Cd. Mx., México.

${ }^{2}$ Universidad Nacional Autónoma de México, Facultad de Estudios Superiores Zaragoza, Herbario FEZA, Batalla de 5 de mayo s/n, colonia Ejército de Oriente, Alcaldía Iztapalapa, 09230 Cd. Mx., México.

${ }^{3}$ Instituto Tecnológico de Ciudad Victoria, Tecnológico Nacional de México, Herbario Micológico José Castillo, Boulevard Emilio Portes Gil No. 1301, 87010 Ciudad Victoria, Tamaulipas, México.

${ }^{4}$ Autor para la correspondencia: traymundoo@ipn.mx; raymundot.tr@gmail.com.
Recibido: 24 de abril de 2020.

Revisado: 29 de mayo de 2020.

Aceptado por Marie-Stéphanie Samain: 7 de julio de 2020.

Publicado Primero en línea: 12 de agosto de 2020.

Publicado: Acta Botanica Mexicana 127 (2020).
Citar como: Sánchez Flores, M., R. Valenzuela, M. A. Hernández-Muñoz, J. García Jiménez, M. Martínez-Pineda y T. Raymundo. 2020. Ascomicetos del bosque mesófilo de montaña de Honey, Puebla de los Ángeles, México. Acta Botanica Mexicana 127: e1719. DOI: 10.21829/abm127.2020.1719 


\section{Introducción}

Los ascomicetos se caracterizan por la formación de ascosporas dentro de ascas derivadas de la reproducción sexual (Alexopoulos y Mins, 1985), se encuentran en numerosos nichos ecológicos y prácticamente en todos los ecosistemas terrestres y marinos. Su principal función es la descomposición de sustratos orgánicos, además de ser mutualistas, parásitos y patógenos de plantas, animales, y de otros hongos (Schoch et al., 2009).

El municipio Honey se localiza al noroeste del estado de Puebla, dentro de la Sierra Norte de Puebla, que a su vez corresponde a la Sierra Madre Oriental. Colinda al norte, sur y oeste con el estado de Hidalgo y al este con el municipio Pahuatlán, en el estado de Puebla. El clima se caracteriza por ser templado húmedo con lluvias abundantes durante el verano y con una temperatura de 7 a $18{ }^{\circ} \mathrm{C}$ durante todo el año (INEGI, 2009). El municipio conserva $47-50 \%$ de su vegetación natural, dentro de la cual podemos encontrar el bosque mesófilo de montaña, con un constante ecotono con bosque de Pinus-Quercus, en un rango altitudinal de 800 hasta 2300 $\mathrm{m}$, pero amenazado por las crecientes actividades humanas (Reyes-Díaz, 2003).

Recientemente, en el área de estudio se citaron Daldinia asphalatum (Link ex Fr.) Sacc., D. childiae J.D. Rogers \& Y.M. Ju, D. eschscholtzii (Ehrenb.) Rehm, D. rehmii Læssøe, M. Stadler \& J. Fourn. (Barbosa-Reséndiz et al., 2020); Pithya cupressina (Batsch) Fuckel (Ortega-López et al., 2019) y Unguiculariopsis ravenelii (Berk. \& M.A. Curtis) W.Y. Zhuang \& Korf (Raymundo et al., 2020). Sin embargo, no existe un estudio exclusivo de los ascomicetos de Honey, por lo que en el presente trabajo se tiene por objetivo conocer las especies del bosque mesófilo de montaña de este municipio y describir los nuevos registros para la micobiota mexicana.

\section{Materiales y Métodos}

Se realizaron recolecciones en temporadas secas y de lluvia, de 2016 a 2018 en distintas localidades del municipio de Honey, Puebla (Fig. 1). Los ejemplares se depositaron en la colección de hongos del Herbario ENCB de la Escuela Nacional de Ciencias Biológicas del Instituto Politécnico Nacional (IPN) y en la colección de hongos del Herbario FEZA de la Facultad de Estudios Superiores de la Universidad Nacional Autónoma de México (UNAM).
El material recolectado se examinó siguiendo las técnicas tradicionales de la micología propuestas por Cifuentes et al. (1986). Los especímenes se caracterizaron en fresco, describiendo los caracteres macroscópicos de acuerdo con su tamaño, forma, color y, en algunos casos, su textura. La terminología hace referencia al diccionario ilustrado de micología (Ulloa y Hanlin, 2006), mientras que los colores se indican según la tabla de color de Kornerup y Wanscher (1978). Después de una rehidratación con alcohol al $70 \%$, se agregó $\mathrm{KOH}$ al 10\%; algunas muestras se tiñeron con reactivo Melzer para observar amiloidia de las ascas. Se observaron, analizaron y caracterizaron las estructuras microscópicas de los diferentes excípulos, paráfisis, ascas y ascosporas, utilizando un microscopio óptico K-7 (Zeiss, Jena, Alemania), los cuales se ilustraron en las descripciones y fotografiaron con una cámara D-7000 y un lente DX Micor 85 mm (Nikon, Tokio, Japón).

Los ejemplares se determinaron utilizando las claves de Denison (1969), Zhuang (1988), San Martín y Rogers $(1989,1993,1995,1996)$, Ju et al. (1998), Rogers y Ju (1998), Hansen et al. (1999), San Martín et al. (1999a, b), Medel y Chacón (2000), Brayford et al. (2004), Calonge et al. (2006), Chaverri et al. (2011) y Tapia et al. (2017).

Los nuevos registros para México, así como la nueva especie se describen macro y micromorfológicamente, para el resto de las especies solo se incluyen notas taxonómicas. La clasificación en este trabajo sigue la de Kirk et al. (2008).

\section{Resultados}

De 106 ejemplares analizados, se determinaron 52 especies, de los cuales 39 son nuevos registros para Puebla de Los Ángeles, mientras que Adelphella babingtoni (Berk. \& Broome) Pfister, Cudoniella acicularis (Bull.) J. Schröt., Lachnum fuscescens (Pers.) P. Karst., Ophioceras leptosporum (S.H. Iqbal) J. Walker, Orbilia curvatispora Boud. y Unguiculariopsis acerina W.Y. Zhuang se describen como nuevos registros para México. Se propone a Hymenoscyphus herrerae Sánchez-Flores, R. Valenz. \& Raymundo como nueva para la ciencia.

Se presenta por primera vez un listado micoflorístico de ascomicetos del bosque mesófilo de la Sierra Norte de Puebla. Se registran 52 especies, pertenecientes a 32 géneros, 23 familias, 11 órdenes y seis clases de Ascomyco- 


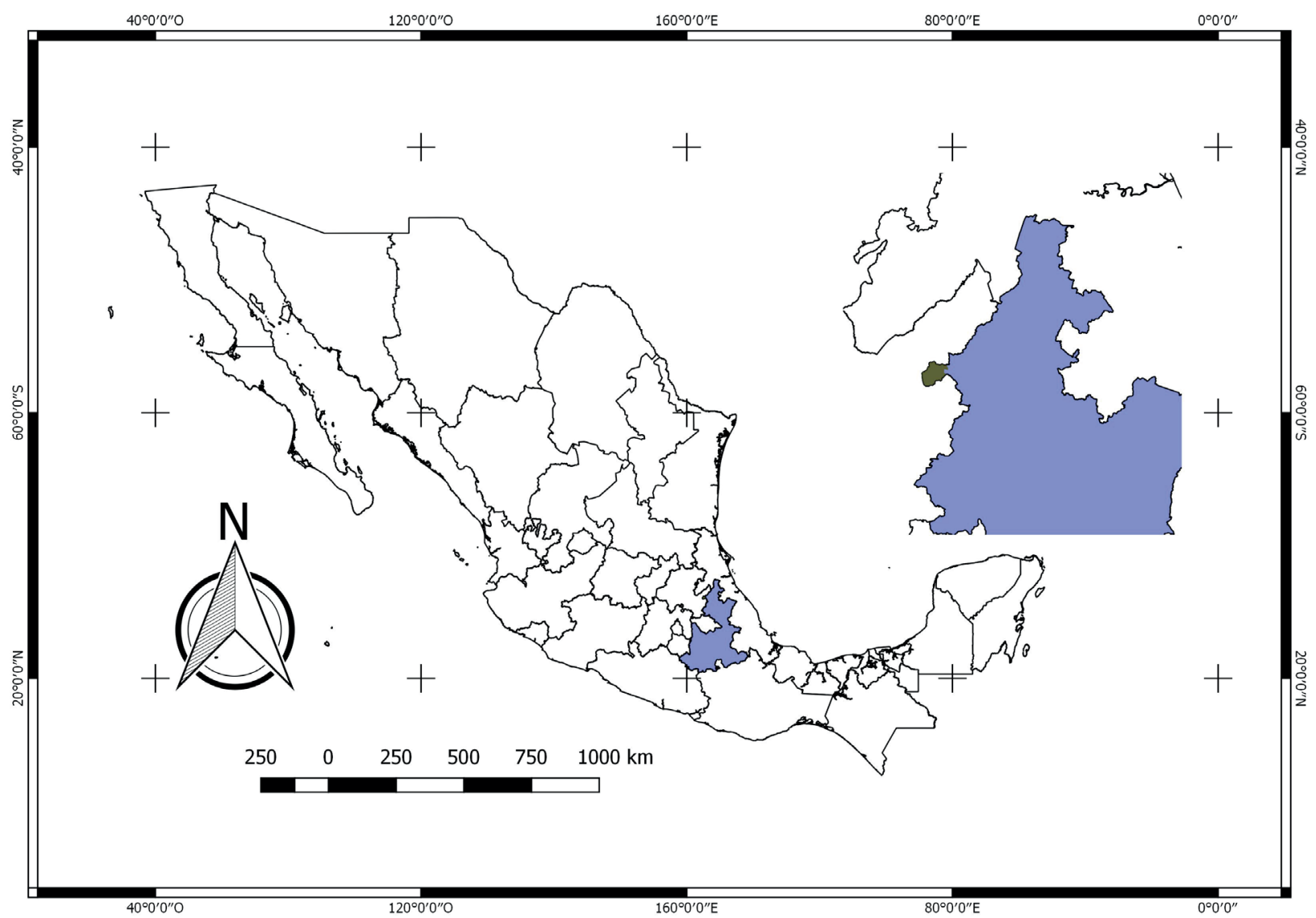

Figura 1: Área de estudio, ubicación geográfica de Honey, Puebla de Los Ángeles, México.

ta. De las 52 especies, 39 se citan por primera vez para el estado de Puebla y seis para el país, señaladas con * y ** respectivamente. La clase que presentó mayor diversidad fue Sordariomycetes con tres órdenes, siete familias, 10 géneros y 26 especies, seguida de Leotiomycetes con tres órdenes, siete familias, ocho géneros y 10 especies, Pezizomycetes con un orden, cinco familias, nueve géneros y 10 especies, Dothideomycetes con dos órdenes, dos familias, dos géneros y dos especies y finalmente Orbiliomycetes con un orden, una familia, dos géneros y tres especies. Cuarenta y una especies, $78.8 \%$ del total, se encontraron creciendo sobre madera, lo cual corresponde con el estudio de los ascomicetos del bosque relicto de Fagus grandifolia Ehrh. subsp. mexicana (Martínez) E. Murray, en el estado de Hidalgo (Raymundo et al., 2019).

Los órdenes representados en el presente estudio con respecto al número de especies de cada uno son Cyttariales (1), Helotiales (8), Hypocreales (6), Leotiales (1), Mag- naporthales (1), Orbiliales (3), Ostropales (1), Patellariales (1), Pezizales (10), Pleosporales (1) y Xylariales (19).

\section{Taxonomía}

Ascomycota

Dothideomycetes

Patellariales

Patellariaceae

Rhytidhysteron rufulum (Spreng.) Speg., Anal. Soc. Cient. Argent. 90(3-6): 177. 1921.

TIPO: PUERTO RICO. Sin localidad, s.f., C. Sprengel s.n. (tipo no localizado). 
$\equiv$ Hysterium rufulum Spreng., K. Svenska Vetensk-Akad. Handl., Ser. 3 41: 50. 1820.

इ Tryblidiella rufula (Spreng.) Sacc., Syll. Fung. (Abellini) 2 757. 1883.

इ Triblidium rufulum (Spreng.) Ellis \& Everh., N. Amer. Pyren. (Newfield): 690. 1892.

इ Brunaudia rufula (Spreng.) Kuntze, Revis. Gen. Pl. (Leipzig) 3(3): 447. 1898.

Hábito: saprobio, lignícola.

Distribución: Oaxaca (Raymundo et al., 2014; Álvarez et al., 2016); Chiapas, Guerrero, Nuevo León, Puebla, Querétaro, Quintana Roo, Sonora, Tabasco, Tamaulipas, Veracruz (Chacón et al., 2014).

Material estudiado: MÉXICO. Puebla, municipio Honey, Cascadas Arcoíris, Rincón de Chila, 20¹5'32.4"N, 98¹4'48.3"O, 26.II.2018, T. Raymundo 7353 (ENCB); loc. cit., 25.XI.2017, M. Sánchez 1102 (ENCB).

Notas taxonómicas: esta especie se caracteriza por histerotecios naviculares, negros, errumpentes, himenio color amarillento-anaranjado, con reacción en $\mathrm{KOH}$ de color magenta; ascas 152-199.5 × 12.2-15 $\mu \mathrm{m}$, cilíndricas; ascosporas $26.6-32.3 \times 5.7-9.5 \mu \mathrm{m}$, elipsoides a alantoides, color marrón, con tres septos transversales.

Pleosporales

\section{Melanommataceae}

*Byssosphaeria rhodomphala (Berk.) Cooke, Grevillea 15(75): 81.1887.

TIPO: ESTADOS UNIDOS DE AMÉRICA. Ohio, sobre madera podrida, s.f., M. J. Berkeley 135 (no se menciona en cual herbario se depositó el espécimen tipo).

इSphaeria rhodomphala Berk., London J. Bot. 4: 313. 1845. इ Melanopsamma rhodomphalos (Berk.) Sacc., Michelia 1 (3): 347. 1878.
इ Herpotrichia rhodomphala (Berk.) Sacc., Syll. Fung. (AbeIlini) 2: 212. 1883.

E Psilosphaeria rhodomphalos (Berk.) Cooke, Grevillea 16 (78): 50. 1887.

Hábito: saprobio, lignícola.

Distribución: Veracruz (Chacón y Tapia-Padilla, 2013). Se cita por primera vez para Puebla.

Material estudiado: MÉXICO. Puebla, municipio Honey, camino a la Cascada Velo de Novia, Chila de Juárez, 20¹7'22.41"N, 98¹3'69.67"O, 21.X.2017, M. Sánchez 1062 (ENCB).

Notas taxonómicas: esta especie se caracteriza por pseudotecios superficiales, solitarios a gregarios, sobre un subículo bien desarrollado, subglobosos a levemente turbinados, poco colapsados; ascas 95-125 × 11-12 $\mu \mathrm{m}$, cilíndricas; ascosporas 19-22 ×6-7 $\mu \mathrm{m}$, fusoides, color marrón, con un septo transversal.

\section{Lecanoromycetes}

\section{Ostropales}

\section{Stictidaceae}

*Stictis radiata (L.) Pers., Observ. Mycol. (Lipsiae) 2: 73. 1800.

TIPO: SUECIA. Sin localidad, sobre madera podrida de abeto, s.f., Linnaeus s.n. (tipo no localizado).

三 Lycoperdon radiatum L., Species Plantarum: 1654. 1763.

三 Chondrioderma radiatumk (L.) Rostaf., Sluzowce Monografia: 182. 1874.

इ Schmitzomia radiata (L.) W. Phillips, A manual of the British Discomycetes: 379. 1887.

इDiderma radiatum (L.) Morgan, Journal of the Cincinnati Society of Natural History 16(2-3): 151. 1894.

Hábito: saprobio, lignícola. 
Distribución: Oaxaca (Raymundo et al., 2013); Hidalgo (Raymundo et al., 2016); Veracruz (López y García, 2002). Se cita por primera vez para Puebla.

Material estudiado: MÉXICO. Puebla, municipio Honey, Cascadas Arcoíris, Rincón de Chila, 20¹5'32.4"N, 98¹4'48.3"O, 26.II.2018, T. Raymundo 7352 (ENCB), 7362 (ENCB); loc. cit., 26.II.2018, M. Sánchez 1153 (ENCB), 1174 (ENCB); loc. cit., 26.Il.2018, R. Valenzuela 18025 (ENCB), 18032 (ENCB).

Notas taxonómicas: esta especie se caracteriza por apotecios inmersos en madera, gregarios, margen recurvado de color blanco y un himenio de color amarillo; ascas 150$180 \times 10-16 \mu \mathrm{m}$, hialinas; ascosporas 140-160 × 1.5-2 $\mu \mathrm{m}$, filiformes, multiseptados.

Leotiomycetes

Cyttariales

Cordieritidaceae

*Unguiculariopsis acerina W.Y. Zhuang, Mycotaxon 32(1): 22. 1988. Figs. 2A-C.

TIPO: ESTADOS UNIDOS DE AMERICA. New York, sobre corteza de Acer rubrum, 11.X.1977, S. H. Strauss s.n. (holotipo: CUP 57020).

Apotecios 385-415 × 152-161 $\mu \mathrm{m}$, discoides, sésiles, margen ondulado, himenio color beige (5D4) cuando fresco y marrón cuando seco, exterior del apotecio marrón grisáceo (5C1), pelos derivados del excípulo de 24-27 × 3-3.5 $\mu \mathrm{m}$, en forma de gancho, adelgazándose en el ápice $0.8 \mu \mathrm{m}$, originándose sobre el excípulo ectal, hialinos, lisos, pared delgada; excípulo ectal 13-23 $\mu \mathrm{m}$ de grosor, textura globosa a angular con células de 4-12 $\mu \mathrm{m}$, globosas, color marrón; excípulo medular textura angular a intrincada, difícil de diferenciar del excípulo ectal; subhimenio 57-60 $\mu \mathrm{m}$; paráfisis 1-2 $\mu \mathrm{m}$, filiformes, hialinas, septadas en la base; ascas 30-38 × 4.5-5 $\mu \mathrm{m}$, clavadas, octosporadas, biseriadas, inamiloides; ascosporas 4-5 × 1.5-2 $\mu \mathrm{m}$, elípticas, hialinas, bigutuladas, pared delgada.
Hábito: epífito, fungícola, crece gregario sobre Orbilia curvatispora y a su vez sobre madera de Quercus sp.

Distribución: Estados Unidos de América (Zhuang, 1988). Se cita por primera vez para México.

Material estudiado: MÉXICO. Puebla, municipio de Honey, Cascadas Arcoíris, 20¹5'32.4"N, 98¹4'48.3"O, 26.II.2018, M. Sánchez 1170 (ENCB).

Notas taxonómicas: crece sobre hongos Corticioides (Zhuang, 1988), pero en este caso se encontró creciendo cerca de Orbilia curvatispora. Se caracteriza por ascas 30$38 \times 4.5-5 \mu \mathrm{m}$, clavadas; ascosporas 4-5 × 1.5-2 $\mu \mathrm{m}$, elípticas, hialinas. Unguiculariposis ravenelii es la única especie del género que se había citado en México.

Helotiales

\section{Chlorociboriaceae}

*Chlorociboria aeruginascens (Nyl.) Kanouse, Mycologia 49(6): 858. 1958.

TIPO: RUSIA. Kolaënsi, sobre madera de Salix, s.f., Nylander s.n. (tipo no localizado).

इ Peziza aeruginascens Nyl., Not. Sällsk. Fauna et Fl. Fenn. Förh., Ny Ser. 10: 42. 1868.

इChlorosplenium aeruginosum var. aeruginascens (Nyl.) P. Karst., Not. Sällsk. Fauna et Fl. Fenn. Förh. 11: 233. 1870. 三Chlorosplenium aeruginascens (Nyl.) P. Karst., Bird. Känn. Finl. Nat. Folk 19: 103. 1871.

इChlorociboria aeruginascens (Nyl.) Kanouse, Mycologia 39(6): 641.1948.

Hábito: saprobio, lignícola en madera.

Distribución: Durango (Raymundo et al., 2012); Guerrero, Morelos, Nuevo León (Valenzuela, 1990); Hidalgo (Frutis y Guzmán, 1983; Raymundo et al., 2019); Sonora (Méndez-Mayboca et al., 2007). Se cita por primera vez para Puebla. 

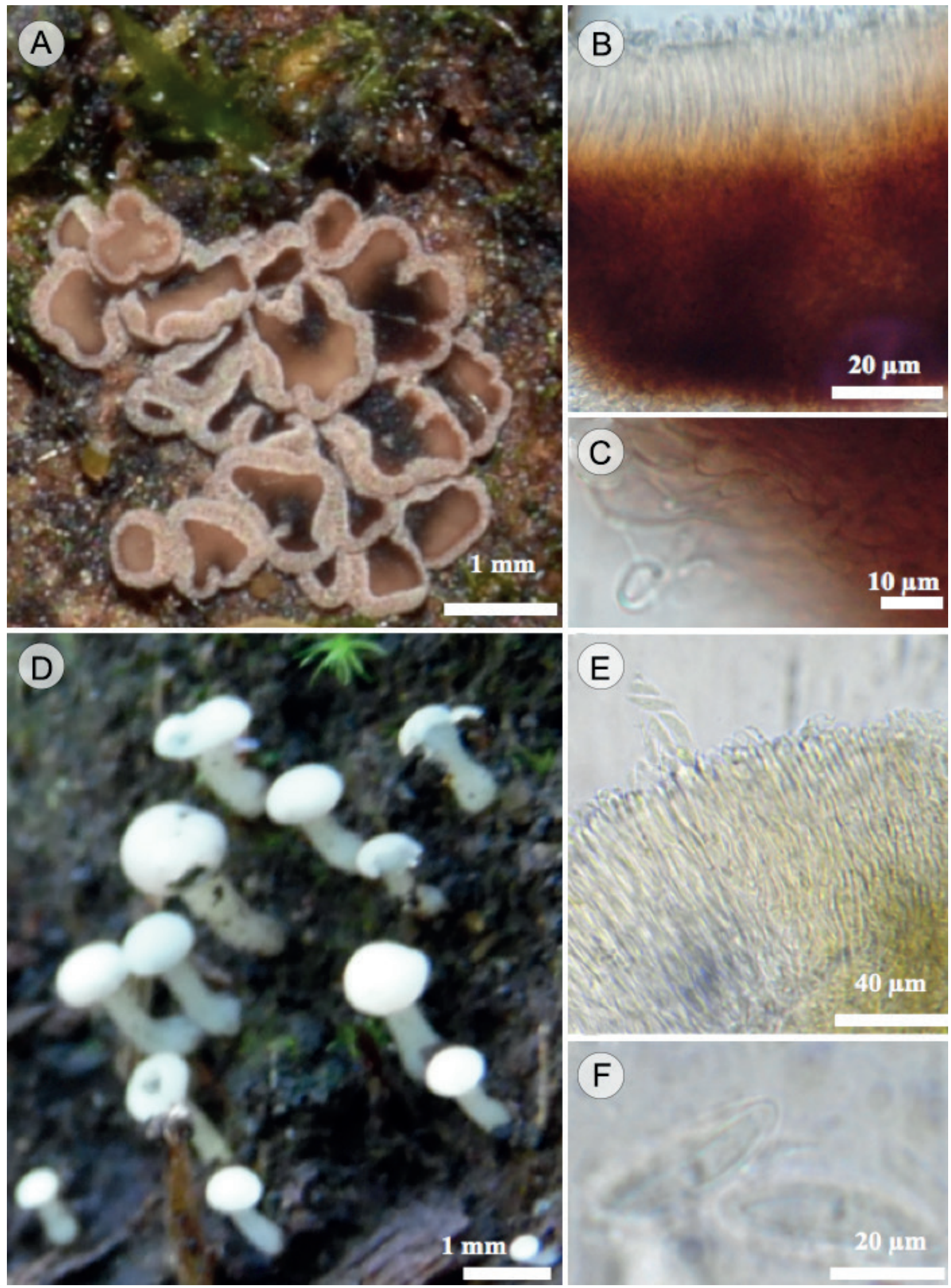

Figura 2: Unguiculariopsis acerina W.Y. Zhuang: A. apotecios; B. corte longitudinal del apotecio; C. pelos ganchudos del excípulo ectal; Cudoniella acicularis (Bull.) J. Schröt.: D. apotecios; E. ascas con ascosporas; F. ascosporas. 
Material estudiado: MÉXICO. Puebla, municipio Honey, Cascadas Arcoíris, Rincón de Chila, 20¹5'32.4" $\mathrm{N}$, $98^{\circ} 14^{\prime} 48.3^{\prime \prime O}, 25 . X I .2017$, S. Rangel 24 (ENCB); loc. cit., 26.II.2018, V. Rodríguez-León 2 (ENCB); loc. cit., M. Sánchez 1090 (ENCB).

Notas taxonómicas: se caracteriza por sus apotecios en forma de copa, espatulados a infundibuliformes, color verde azulado; ascas 45-50 × 3-4 $\mu \mathrm{m}$, cilíndricas-clavadas, amiloides; ascosporas 4.8-6.4 $\times$ 0.8-1.5 $\mu \mathrm{m}$, fusiformes, cilíndricas a alantoides, hialinas.

\section{Helotiaceae}

Hymenoscyphus herrerae Sánchez-Flores, R. Valenz. \& Raymundo, sp. nov. Fig. 3.

TIPO: MÉXICO. Puebla de Los Ángeles, municipio Honey, Cascadas Arcoíris, Rincón de Chila, 20¹5'32.4" N, 98¹4'48.3"O, 26.II.2018, T. Raymundo 7364 (holotipo: ENCB!).

Apothecia 4-8 mm longa, 4-6 mm latum, ascis 90-95 $\times$ 7.6-9.5 $\mu \mathrm{m}$, clavati, hyalinis, uniseriate, octosporicas; ascosporae 27-47 × 3-6 $\mu \mathrm{m}$, fusiforms, lux brunneis, 5-7 septata, septis transversis divisae.

Apotecios estipitados, 4-8 $\mathrm{mm}$ de alto, $4-6 \mathrm{~mm}$ de diámetro, solitarios a gregarios, color amarillo (30A7), himenio liso; estípite liso, 2-4 mm de largo, central, filiforme; excípulo ectal 44-48 $\mu \mathrm{m}$ de grosor, textura porrecta, formado por células de 4.8-5.6 $\mu \mathrm{m}$; excípulo medular 45-50 $\mu \mathrm{m}$ de grosor, formado por hifas de textura intrincada; himenio 105-127 $\mu \mathrm{m}$ de grosor, paráfisis de $3 \mu \mathrm{m}$ de diámetro, filiformes, septadas; ascas 90-95 × 7.6-9.5 $\mu \mathrm{m}$, claviformes, octosporadas, uniseriadas, con poro apical amiloide; ascosporas 27-47 × 3-6 $\mu \mathrm{m}$, fusiformes con extremos agudos, hialinas, pared delgada y lisa, con 5-7 septos.

Hábito: saprobio, lignícola, crece sobre madera.

Distribución: conocido únicamente del municipio de la localidad tipo.
Etimología: dedicado al Dr. Teófilo Herrera (19242020) por sus aportaciones a la Micología.

Material estudiado: MÉXICO. Puebla, municipio de Honey, Parque Turístico El Sabinal, 20¹4'05.1"N, 98¹2'49.8"O, 17.XI.2016, M. Sánchez 747 (ENCB, FEZA); San Pedro Chila, 20¹7'38.78"N, 98¹3'24.69"O, 17.IX.2017, M. Sánchez 949 (ENCB), 951 (ENCB), 954 (ENCB), 955 (ENCB), 958 (ENCB); Cascada Velo de Novia, 20¹7'22.41"N, 98¹3'69.67"O, 21.X.2017, M. Sánchez 1053 (ENCB); loc. cit., 22.X.2017, M. Sánchez 1074 (ENCB); Cascadas Arcoíris, Rincón de Chila, 20¹5'32.4"N, 98¹4'48.3"O, 25.XI.2017, M. Sánchez 1103 (ENCB); carretera Acahuales - Chila, 20¹5'45.3"N, 98¹1'58.3"O, 26.XI.2017, M. Sánchez 1115 (ENCB), 1117 (ENCB).

Notastaxonómicas: unaespecieafines Hymenoscyphus scutula (Pers.) Phillips; sin embargo, se diferencian porque las ascosporas son de $18-27 \times 3.5-5 \mu \mathrm{m}$ de acuerdo con Dennis (1981) en especímenes de Gran Bretaña. Hengstmengel (1996) reporta un rango de 20-27 × 3-4 $\mu \mathrm{m}$ para Holanda, y Uzun et al. (2014) citaron una talla de 19-25 × 3.5-4.5 $\mu \mathrm{m}$ para especímenes de Turquía. Macroscópicamente, $H$. scutula y $H$. herrerae presentan una morfología similar.

\section{Lachnaceae}

*Incrucipulum ciliare (Schrad.) Baral, Beih. Z. Mykol. 6: 72. 1985.

TIPO: GALES. Sobre troncos de madera de Haya, s.f., Scharder s.n. (espécimen tipo no designado, ni se menciona en cual que herbario se depositó).

E Peziza ciliaris Schard., J. Bot. (Schrader) 2(1): 63. 1799.

三 Hyalopeziza ciliaris (Schard.) Fuckel, Jb. Nassau. Ver. Naturk. 23-24: 298. 1870.

इ Lachnea ciliaris (Schard.) Gillet, Champignons de France, Discom. 3: 68. 1880.

三 Trichopeziza ciliaris (Schard.) Rehm, Berichte des Naturhistorischen Vereins Augsburg 26: 64. 1881.

इErinella ciliaris (Schard.) Quél., Enchir. Fung. (Paris): 304. 1886. 

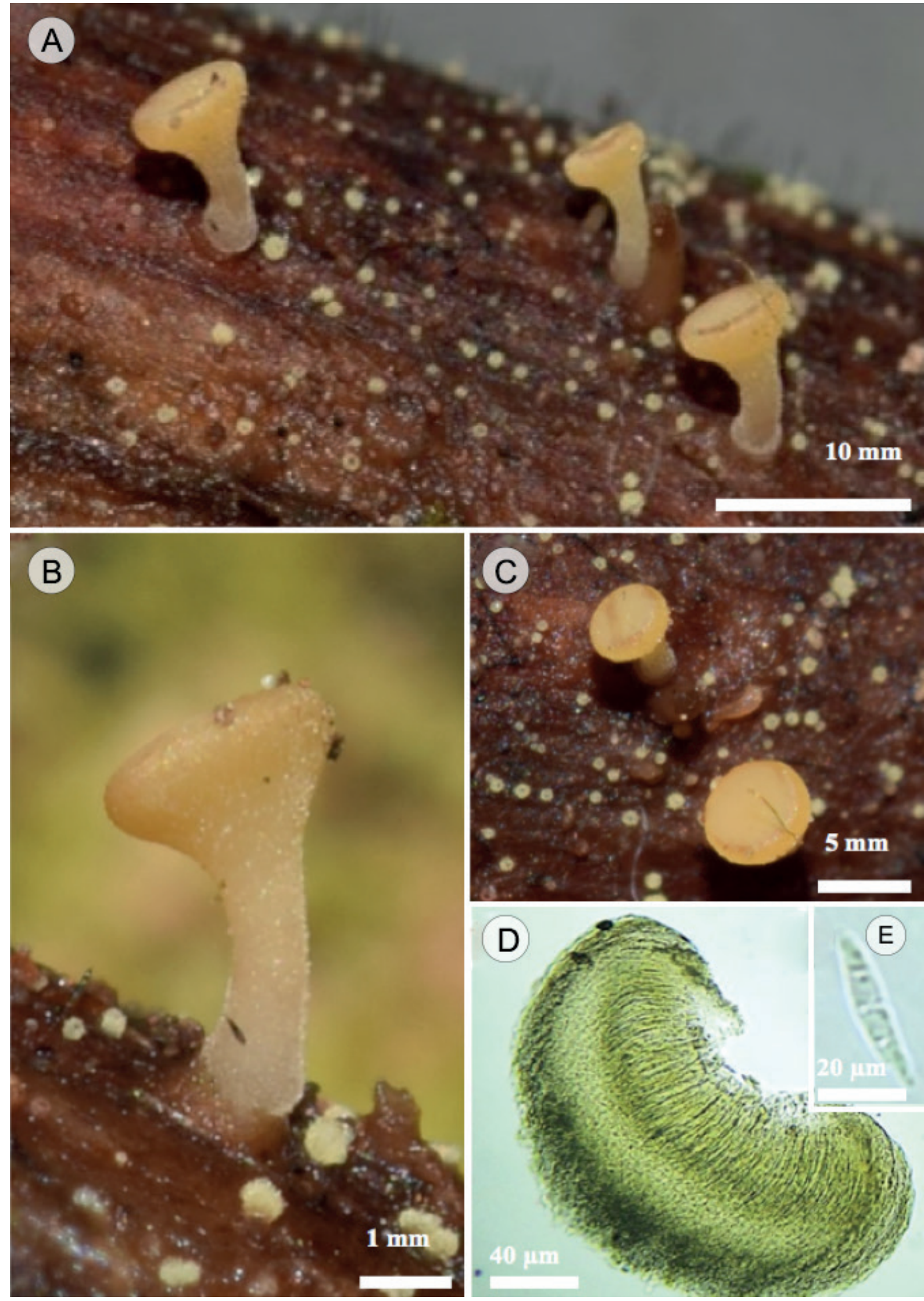

Figura 3: Hymenoscyphus herrerae Sánchez-Flores, R. Valenz. \& Raymundo: A, B. apotecios; C. apotecios mostrando himenio; D. corte longitudinal del apotecio; E. ascospora. 
三 Lachnella ciliaris (Schard.) W. Phillips, Man. Brit. Discomyc. (London): 251. 1887.

इ Lachnum ciliare (Schard.) Rehm, Rabenh. Krypt.-Fl., Edn 2 (Leipzig) 1.3(41): 877. 1893.

Hábito: saprobio, lignícola, sobre Cyathea fulva (M. Martens \& Galeotti) Fée.

Distribución: Hidalgo (Raymundo et al., 2016). Se cita por primera vez para Puebla.

Material estudiado: MÉXICO. Puebla, municipio Honey, Cascadas Arcoíris, Rincón de Chila, 20¹5'32.4' $\mathrm{N}$, 98 $141^{\prime} 48.3^{\prime}$ O, 26.II.2018, T. Raymundo 7368 (ENCB); loc. cit., 26.II.2018, R. Valenzuela 18039 (ENCB).

Notas taxonómicas: esta especie se caracteriza por apotecios discoides cubierto de pelos blancos, con un estípite central, himenio de color blanco a pálido amarillento; ascas 60-80 × 5-6 $\mu \mathrm{m}$; ascosporas 18-24 × 2-2.4 $\mu \mathrm{m}$, cilíndricas a fusiformes, hialinas.

*Lachnum apalum (Berk. \& Broome) Nannf., Svensk bot. Tidskr. 30(3): 299. 1936.

TIPO: GRAN BRETAÑA. Spye Park, Batheaston, sobre madera muerta, II.1850, Berkeley y Broome s.n. (no se menciona en cual herbario se depositó el espécimen tipo).

三 Peziza apala Berk. \& Broome, Ann. Mag. Nat. Hist., Ser. 2.7: 180.1851.

इ Lachnella apala (Berk. \& Broome) W. Phillips, Man. Brit. Discomyc. (London): 253. 1887.

इ Erinella apala (Berk. \& Broome) Sacc. (as hapala), Syll. Fung. (Abellini) 8: 509. 1889.

इ Dascycyphus apalus (Berk. \& Broome) Dennis (as Dasyscypha apala), Mycol. Pap. 32: 25. 1949.

Hábito: saprobio, lignícola.

Distribución: Hidalgo (Raymundo et al., 2016). Se cita por primera vez para Puebla.
Material estudiado: MÉXICO. Puebla, municipio Honey, Parque Turístico El Sabinal, $20^{\circ} 14^{\prime} 05.1^{\prime \prime} \mathrm{N}, 98^{\circ} 12^{\prime} 49.8^{\prime \prime O} \mathrm{O}$, 17.XI.2016, M. Sánchez 758 (ENCB, FEZA); camino a la Cascada Velo de Novia, Chila de Juárez, 20¹7'22.41"N, 98¹3'69.67"O, 21.X.2017, M. Sánchez 1061 (ENCB); Cascadas Arcoíris, Rincón de Chila, 20¹5'32.4"N, 98¹4'48.3"O, 26.II.2018, M. Sánchez 1141 (ENCB), 1142 (ENCB), 1145 (ENCB).

Notas taxonómicas: esta especie se caracteriza por apotecios cupuliformes de color blanco, pelos creciendo del excípulo color blanco, himenio color amarillo liso; ascas 90-110 × 6-8 $\mu \mathrm{m}$, cilíndricas, con poro amiloide; ascosporas $33.5-60 \times 1.6 \mu \mathrm{m}$, filiformes, hialinas.

**Lachnum fuscescens (Pers.) P. Karst., Acta Soc. Fauna Flora Fenn. 2(6): 134. 1885. Fig. 4.

TIPO: FINLANDIA. Sin localidad, sobre hojas podridas, s.f., Persoon s.n. (tipo no localizado).

इ Peziza fuscescens Pers., Syn. meth. fung. (Göttingen) 2: 654. 1801.

इ Dasyscyphus fuscescens (Pers.) Gray, Nat. Arr. Brit. PI. (London) 1: 671. 1821.

इ Lachnella fuscescens (Pers.) W. Phillips, Man. Brit. Discomyc. (London): 235. 1887.

इ Atractobolus fuscescens (Pers.) Kuntze, Revis. Gen. PI. (Leipzig) 3(3): 445. 1898.

= Dasyscyphus fuscescens var. fagicola (W. Phillips) Dennis, (as "Dasyscypha"), Mycol. Pap. 32: 38. 1949.

इ Brunnipila fuscescens (Pers.) Baral, Beih. Z. Mycol. 6: 50. 1985.

Apotecios estipitados, 2-5 mm de alto, $4-5 \mathrm{~mm}$ de diámetro, solitarios a gregarios, himenio color blanco, liso, exterior del apotecio cubierto de pelos color marrón (5D7) creciendo del excípulo ectal, 80-124 × 4-5 $\mu \mathrm{m}$, septados, con cristales incrustados y ápice capitado; excípulo ectal 13.6-21.6 $\mu \mathrm{m}$ de grosor, textura globulosa 6.4-88 × 4-7.2 $\mu \mathrm{m}$, a prismática a los extremos, $11-24 \times 5.6-10 \mu \mathrm{m}$, formado por células hialinas de pared delgada; excípulo medular 21-161 $\mu \mathrm{m}$, adelgazando hacia el margen, textura intrin- 

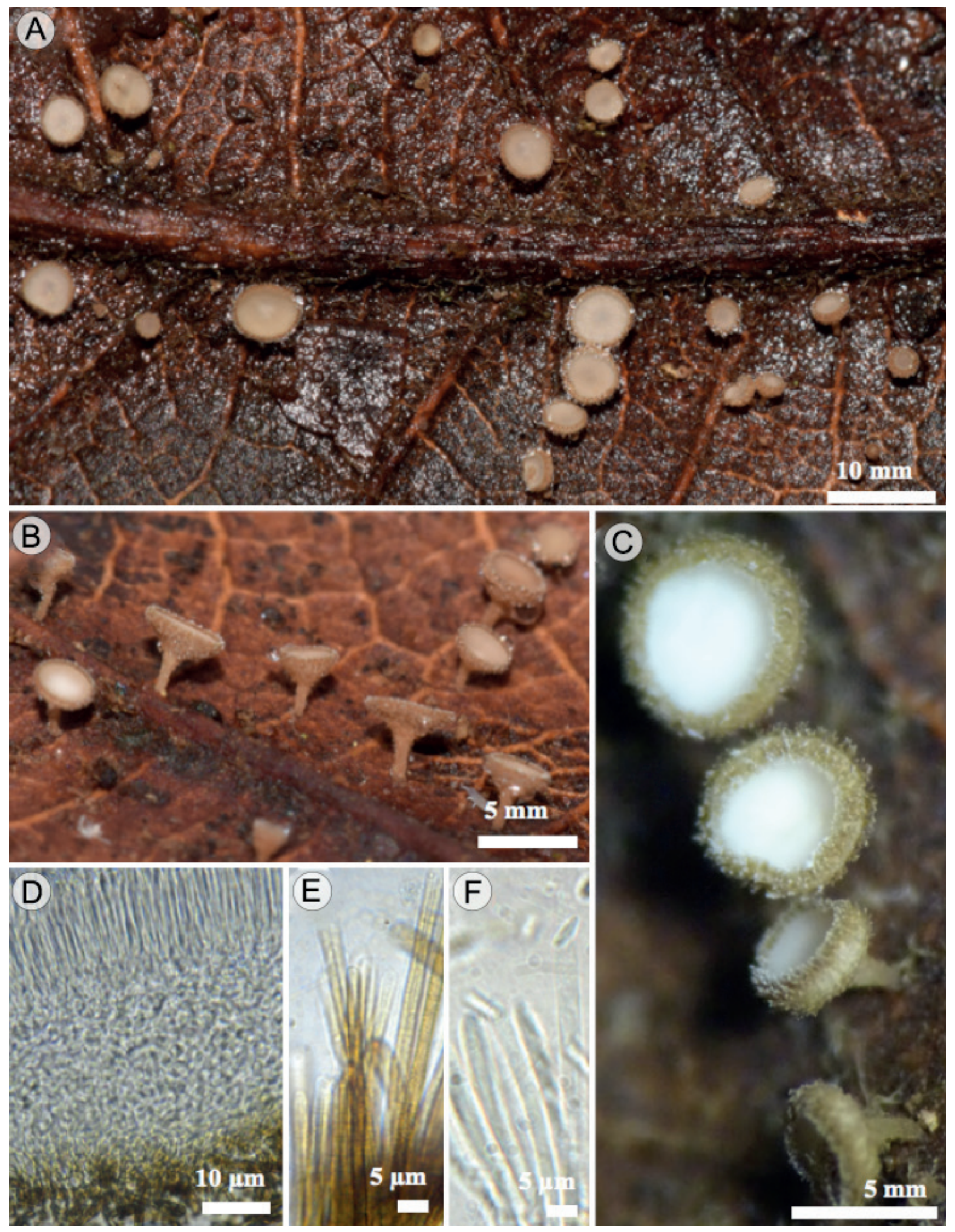

Figura 4: Lachnum fuscescens (Pers.) P. Karst.: A-B; apotecios; C. apotecios mostrando himenio; D. corte longitudinal del apotecio; E. pelos; F. ascas con ascosporas. 
cada de hifas hialinas de 2-3 $\mu \mathrm{m}$; subhimenio 44-56 $\mu \mathrm{m}$; excípulo himenial 44-52 $\mu \mathrm{m}$; paráfisis 3-4 $\mu \mathrm{m}$, lanceoladas, filiformes, hialinas; ascas 40-45 × 4-5 $\mu \mathrm{m}$, cilíndricas, clavadas, octosporadas, biseriadas; ascosporas 5.5-7 × 1-1.6 $\mu \mathrm{m}$, cilíndricas a fusiformes, hialinas, lisas.

Hábito: saprobio, humícola, crece sobre hojarasca.

Distribución: Finlandia (Persoon, 1801); Inglaterra (Dennis, 1981); Suiza (Breitenbach y Kränzlin, 1984). Se cita por primera vez para México.

Material estudiado: MÉXICO. Puebla, municipio Honey, Cascadas Arcoíris, 20¹5'32.4"N, 98¹4'48.3"O, 26.II.2018, T. Raymundo 7359 (ENCB); loc. cit., 26.II.2018, M. Sánchez 1172 (ENCB); loc. cit., 26.II.2018, R. Valenzuela 18059 (ENCB).

Notas taxonómicas: se caracteriza por apotecios estipitados color marrón; ascas 40-45 × 4-5 $\mu \mathrm{m}$, cilíndricas, con poro amiloide; ascosporas 5.5-7 × 1-1.6 $\mu \mathrm{m}$, cilíndricas a fusiformes, hialinas, lisas.

*Lachnum virgineum (Batsch) P. Karst., Bidr. Känn. Finl. Nat. Folk 19: 169. 1871.

इ Peziza virginea Batsch, Elench. Fung. (Halle): 125. 1783. TIPO: FINLANDIA. Mustiala, sobre madera, s.f., Batsch s.n. (Espécimen tipo no designado, ni se menciona en cual herbario se depositó).

三 Dasyscyphus virgineus (Batsch) Gray (as Dasyscypha), Nat. Arr. Brit. PI. (London) 1: 671. 1821.

= Cyathicula virginea (Batsch) P. Karst., Enum. Fungorum et Myxomycetum in Lappio Orientali: 207. 1866.

三Erinella virginea (Batsch) Quél., Enchir. Fung. (Paris): 304. 1886.

इ Lachnella virginea (Batsch) W. Phillips, Man. Brit. Discomyc. (London): 248. 1887.

इ Atractobolus virgineus (Batsch) Kuntze, Revis. Gen. PI. (Leipzig) 3(3): 445. 1898.

Hábito: saprobio, humícola en hojarasca de Quercus sp.
Distribución: Baja California (Galán et al., 1994), Hidalgo (Raymundo et al., 2019), Oaxaca (Raymundo et al., 2013). Se cita por primera vez para Puebla.

Material estudiado: MÉXICO. Puebla, municipio Honey, Cascadas Arcoíris, Rincón de Chila, 20¹5'32.4"N, 98¹4'48.3"O, 26.II.2018, T. Raymundo 7360 (ENCB); loc. cit., 26.II.2018, M. Sánchez 1173 (ENCB); loc. cit., 26.II.2018, R. Valenzuela 18030 (ENCB).

Notas taxonómicas: esta especie se caracteriza por apotecios solitarios a gregarios, color blanco, cubierto de pelos color blanco, estipitados; ascas 45-60 × 4-5 $\mu \mathrm{m}$, cilíndricas, con poro amiloide; ascosporas 5-9 $\times 1-2 \mu \mathrm{m}$, fusiformes, hialinas.

Mollisiaceae

*Mollisia cinerea (Batsch) P. Karst., Bidr. Känn. Finl. Nat. Folk 19: 189. 1871.

इ Peziza cinerea Batsch, Elench. Fung. Cont. Prim. (Halle): 97. 1786. TIPO: RUSIA. Kamtschatka, sobre árboles y ramas en descomposición, s.f., Batsch s.n. (espécimen tipo no designado, ni se menciona en cual herbario se depositó).

इ Octospora cinerea (Batsch) Gray, Nat. Arr. Brit. PI. (London) 1: 667. 1821.

इ Niptera cinerea (Batsch) Fuckel, Jb. Nassau. Ver. Naturk. 23-24: 292. 1870.

Hábito: saprobio, lignícola, creciendo sobre madera en descomposición de Quercus sp.

Distribución: Hidalgo (Raymundo et al., 2019). Se cita por primera vez para Puebla.

Material examinado: MÉXICO. Puebla, municipio Honey, carretera Acahuales - Chila, Chila de Juárez, 20¹5'45.3"N, 98¹1'58.3"O, 26.XI.2017, M. Sánchez 1119 (ENCB).

Notas taxonómicas: esta especie se caracteriza por apotecios discoides, lobulados a irregulares, color cinéreo, 
gris azulado a gris opaco; ascas 60-72 $\times 4-4.8 \mu \mathrm{m}$, octosporadas, cilíndricas, hialinas; ascosporas (6.4)7.2-9.6 × 1.6-2.4 $\mu \mathrm{m}$, cilíndricas a fusoides, biseriadas, hialinas, lisas.

\section{Tricladiaceae}

**Cudoniella acicularis (Bull.) J. Schröt., in Cohn, Kript.-Fl. Schlesien (Breslau) 3.2(1-2): 21. 1983. Figs. 2D-F.

三 Helvella acicularis Bull., Herb. Fr. (Paris) 10: tab. 473, fig. 1. 1790. TIPO: FRANCIA. Paris, sobre troncos podridos de Quercus, s.f., P. Builliard s.n. (Herb. France, tipo no localizado).

इ Leotia acicularis Pers., Observ. mycol. (Lipsiae) 2: 20. 1800. 1799.

इ Helotium aciculare (Bull.) Pers., Dyn. meth. fung., (Göttingen) 2: 677. 1801.

इ Peziza acicularis (Bull.) Fr., Syst. mycol. (Lundae) 2(1): 156.

1822.

इ Sarea acicularis (Bull.) Schwein., Trans. Am. Phil. Soc., New Series 4(2): 178. 1832. 1834.

Apotecio 1-3 mm, en la cabeza himenial, color blanco, con un estípite 1-3 mm de largo, textura gelatinosa-carnosa; excípulo ectal 38-81.7 $\mu \mathrm{m}$, textura prismática constante en todo el hongo, células hialinas; excípulo medular 197.6$246.4 \mu \mathrm{m}$, textura intrincada, con hifas hialinas 2.4-4 $\mu \mathrm{m}$; excípulo himenial 104-127 $\mu \mathrm{m}$; paráfisis 3-5 $\mu \mathrm{m}$, filiformes, hinchados en el ápice, hialinos; ascas 80-120 × 10-13 $\mu \mathrm{m}$, octosporadas, cilíndricas-clavadas, inamiloides, ápices ligeramente lanceolados; ascosporas (12.8)15.2-19.2 × 4-4.8 $\mu \mathrm{m}$ fusiformes, lisas, pared delgada, hialinas, biseriadas.

Hábito: saprobio, lignícola, sobre madera.

Distribución: Francia (Builliard, 1790); Estados Unidos de América (Beug et al., 2014); Inglaterra (Dennis, 1981); Suiza (Breitenbach y Kränzlin, 1984). Se cita por primera vez para México.

Material estudiado: MÉXICO. Puebla, municipio Honey, Cascadas Arcoíris, 20¹5'32.4"N, 98¹4'48.3"O, 25.XI.2017, M. Sánchez 1086 (ENCB).
Notas taxonómicas: Kučera (2011) menciona que $C$. acicularis presenta esporas de 19-22 × 4-5 $\mu \mathrm{m}$ en ejemplares europeos, mientras que Beug et al. (2014) indican medidas de 15-22 × 4-5 $\mu \mathrm{m}$ en especímenes de Estados Unidos de América, similar al material estudiado de México.

\section{Leotiales}

\section{Leotiaceae}

Leotia lubrica (Scop.) Pers., Comm. fung. clav. (Lipsiae): 31. 1797.

इ Helvella lubrica Scop., Fl. Carniol., Edn. 2 (Wien) 2: 477. 1772. TIPO: FRANCIA. En el bosque, s.f., Scopoli s.n. (espécimen tipo no designado, ni se menciona en cual herbario se depositó).

三 Peziza cornucopiae Hoffm., Vegetabilia Cryptogama 2: 21. 1790.

= Leotia viscosa Fr., Syst. Mycol. (Lundae) 2(1): 30. 1822. TIPO: ESTADOS UNIDOS DE AMÉRICA. Carolina del Sur, s.f., Fries s.n. (tipo no localizado).

Hábito: micorrizógeno, terrícola.

Distribución: Veracruz, Oaxaca (Welden y Guzmán, 1978), Hidalgo (Varela y Cifuentes 1979; Frutis y Guzmán, 1983; Raymundo et al., 2019), Nuevo León (Castillo et al., 1979; Garza et al., 1985), Puebla, Coahuila, Ciudad de México, Durango, Morelos, Nuevo León, Estado de México y Veracruz (Chacón y Guzmán, 1983), Morelos (Bautista et al., 1986), Estado de México (Chio et al., 1988), Michoacán (Cifuentes et al., 1990); Sonora (Pérez-Silva et al., 1996), Jalisco (Herrera et al., 2002), Guerrero, Querétaro, Nayarit, Tlaxcala (Ramírez-López y Villegas-Ríos, 2007), Durango (Raymundo et al., 2012); Oaxaca (Raymundo et al., 2013).

Material estudiado: MÉXICO. Puebla, municipio Honey, camino a la Cascada Velo de Novia, Chila de Juárez, 20¹7'22.41"N, 98¹3'69.67"O, 21.X.2017, M. Sánchez 1052 (ENCB).

Notas taxonómicas: esta especie se caracteriza por ascomas estipitados, cespitosos, gelatinosos, color verde 
oliváceo; ascas 115-150 × 7-10 $\mu \mathrm{m}$, cilíndricas, amiloides; ascosporas 15-21 $\times$ 4-5.7 $\mu \mathrm{m}$, fusoides a estrechamente elipsoides, hialinas, 3-5 septos. En Nayarit, Oaxaca y Sonora citada como L. viscosa López-Ríos, actualmente sinónimo de L. lubrica (Index Fungorum, 2020).

\section{Orbiliomycetes}

\section{Orbiliales}

\section{Orbiliaceae}

*Hyalorbilia inflatula (P. Karst.) Baral \& G. Marson, Micologia 2000 (Trento): 44. 2001.

इ Peziza inflatula P. Karst., Not. Sällsk. Fauna et Fl. Fenn. Förh. 10: 175. 1869. TIPO. FINLANDIA. Mustiala, sobre corteza y madera de árboles, principalmente Populus, s.f., P. Karsten s.n. (no se menciona en cual herbario se depositó el espécimen tipo).

三Orbilia inflatula (P. Karst.) P. Karst., Bidr. Känn. Finl. Nat. Folk 19: 108. 1871.

三 Calloria inflatula (P. Karst.) W. Phillips, Man. Brit. Discomyc. (London): 335. 1887.

इ Hyalina inflatula (P. Karst.) Boud., Hist. Class. Discom. Eur. (Paris): 104. 1907.

Hábito: epífito, crece sobre madera en descomposición de Quercus sp.

Distribución: Finlandia (Karsten, 1869); Veracruz (López y García, 2010), Hidalgo (Raymundo et al., 2016; 2019). Se cita por primera vez para Puebla.

Material estudiado: MÉXICO. Puebla, municipio Honey, riachuelo cerca del Río San Marcos, San Pedro Chila, 20¹7'38.78"N, 98¹3'24.69"O, 17.IX.2017, M. Sánchez 953 (ENCB); camino a la Cascada Velo de Novia, Chila de Juárez, 20¹7'22.41"N, 98¹3'69.67"O, 21.X.2017, M. Sánchez 1060 (ENCB).

Notas taxonómicas: esta especie se caracteriza por apotecios sésiles en forma de copa a discoides, color hialino amarillento, delgado y translúcido; ascas 24-30 × 3-4.5 $\mu \mathrm{m}$, cilíndricas; ascosporas 4-6 × 0.6-1 $\mu \mathrm{m}$, subcilíndricas, hialinas.

**Orbilia curvatispora Boud., Bull. Soc. Mycol. Fr. 4(2): 80 . 1888. Figs. 5A-B.

TIPO: FRANCIA. Cerca de Montmorency, sobre corteza podrida de Quercus, VII.1885, Boudier s.n. (no se menciona en cual herbario se depositó el espécimen tipo).

Apotecios 1-1.5 mm de diámetro, solitarios a gregarios, cerosos, discoide a cupuliformes poco profundos, translúcidos cuando están frescos, volviéndose opacos al secarse, superficie externa color blanco a amarillo intenso (30A4) o naranja (5A5), lisa; superficie himenial rosa, cóncava, lisa; margen entero a ligeramente ondulado; excípulo ectal 45.6$89.6 \mu \mathrm{m}$, células angulares 7-25 × 6.4-16.8 $\mu \mathrm{m}$ de diámetro, hialinas; excípulo medular 20-28 $\mu \mathrm{m}$ de grosor, textura intrincada; himenio 25.6-28 $\mu \mathrm{m}$; paráfisis $1.2 \mu \mathrm{m}$, filiformes, hialinas, no septadas, ramificadas en la base, ápices abruptamente hinchados de hasta $4.5 \mu \mathrm{m}$ de diámetro; ascas 20-25 $\times 2-3.8 \mu \mathrm{m}$, cilíndrico-clavadas, octosporadas, amiloides, con los ápices estrechos pero obtusos; ascosporas 7.2-8 × $1 \mu \mathrm{m}$, fusoides a alantoides, hialinas, paredes delgadas y lisas, unicelulares con una gútula.

Hábito: epífito, creciendo sobre madera en descomposición de Quercus sp.

Distribución: Francia (Boudier, 1888); India, Rusia, Venezuela (Thind y Sharma, 1980); Inglaterra (Dennis, 1981); Suiza (Breitenbach y Kränzlin, 1984). Se cita por primera vez para México.

Material estudiado: MÉXICO. Puebla, municipio Honey, Cascadas Arcoíris, 20¹5'32.4"N, 98¹4'48.3"O, 26.II.2018, M. Sánchez 1169 (ENCB).

Notas taxonómicas: esta especie se caracteriza por apotecios discoides color blanco, amarillo o naranja; ascas 20$25 \times 2-3.8 \mu \mathrm{m}$, cilíndricas, poro apical amiloide, ápices obtusos; ascosporas 7.2-8 $\times 1 \mu \mathrm{m}$, fusoides a alantoides, hialinas. 

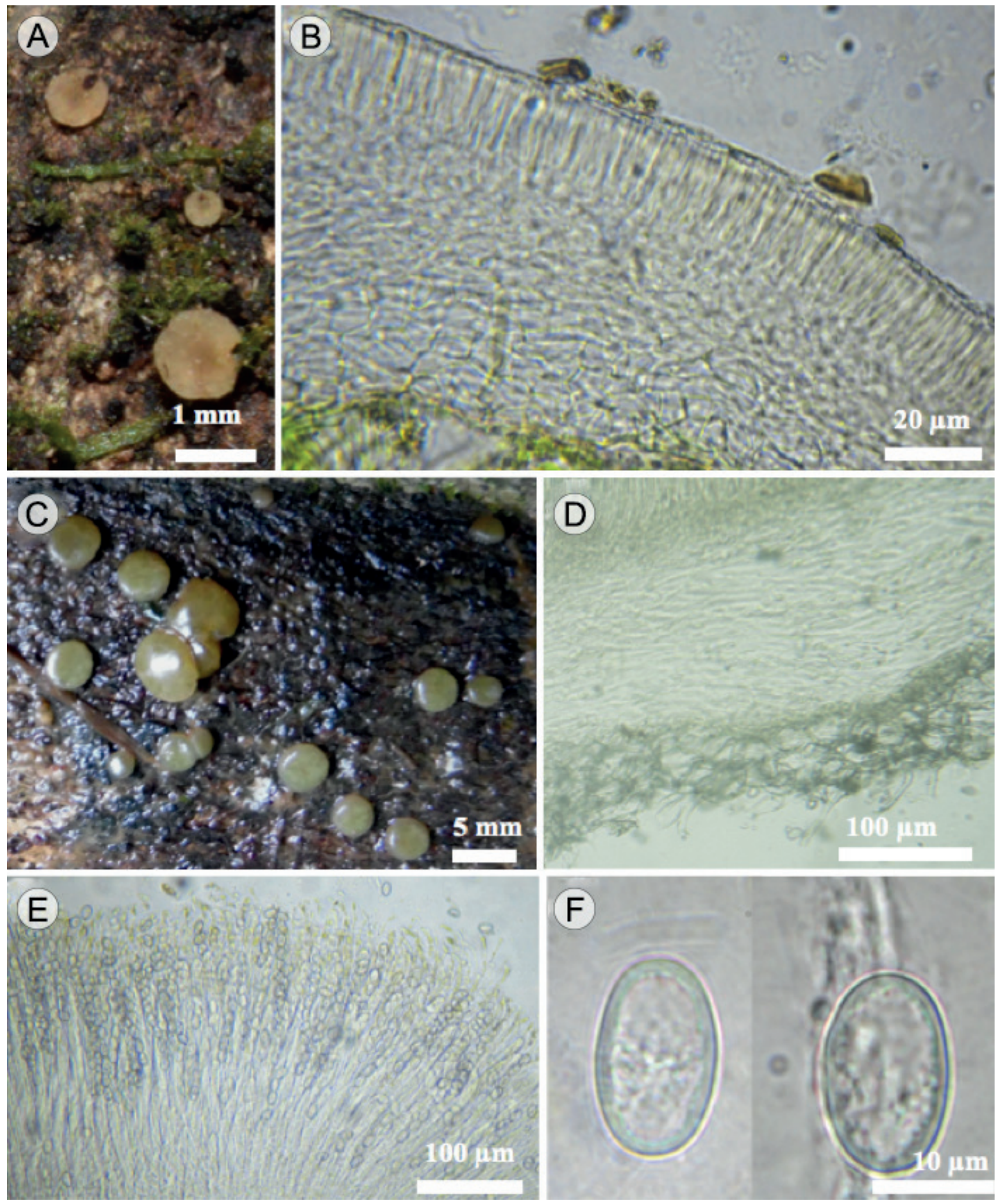

Figura 5: Orbilia curvatispora Boud.: A. apotecios; B. corte longitudinal del apotecio; Adelphella babingtonii (Berk. \& Broome) Pfister, Matočec \& I. Kušan: C. apotecios; D. corte longitudinal del apotecio; E. ascas con ascosporas; F. ascosporas. 
*Orbilia xanthostigma (Fr.) Fr., Summa veg. Scand., Sectio Post. (Stockholm): 357. 1849.

三 Peziza xanthostigma Fr., Observ. Mycol. (Havniae) 1: 166. 1815. TIPO: POLONIA. Sin localidad, sobre madera de pino podrido, s.f., Fries s.n. (tipo no localizado).

三 Mollisia xanthostigma (Fr.) Gillet, Champignons de France, Dicom (5): 125. 1882.

三 Calloria xanthostigma (Fr.) W. Phillips, Ma. Brit. Discomyc. (London): 329. 1887.

Hábito: epífito, crece sobre madera en descomposición de Quercus sp.

Distribución: Ciudad de México, Oaxaca (Chacón y Guzmán, 1983); Hidalgo (Raymundo et al., 2019). Se cita por primera vez para Puebla.

Material estudiado: MÉXICO. Puebla, municipio Honey, Cascadas Arcoíris, Rincón de Chila, 20¹5'32.4"N, 98¹4'48.3"O, 26.II.2018, M. Sánchez 1163 (ENCB), 1167 (ENCB).

Notas taxonómicas: esta especie se caracteriza por apotecios de 1-1.5 mm de diámetro, subsésiles, discoides, color amarillo cuando fresco; ascas 25-38 × 2.5-4.2 $\mu \mathrm{m}$, cilíndricas, poro apical amiloide, ápices truncados; ascosporas 2-3 × 1-1.5 $\mu \mathrm{m}$, ligeramente alantoides, hialinas.

\section{Pezizomycetes}

Pezizales

Helvellaceae

*Helvella solitaria P. Karst., Bird. Känn. Finl. Folk 19: 37. 1871.

इ Peziza solitaria P. Karst., Not. Sällsk. Fauna et Fl. Fenn. Förh. 10: 111. 1869. TIPO: SUECIA. Sobre tierra homosa-arenosa en los meses de septiembre, s.f., Karsten 1832 (holotipo: H). 三 Lachnea solitaria Bizz. \& Sacc., Mycotheca Veneti 4: no. 323. 1876
Hábito: micorrizógeno, terrícola.

Distribución: Ciudad de México (Vite-Garín et al., 2006), Jalisco (Landeros y Guzmán-Dávalos, 2013) y Oaxaca (Raymundo et al., 2013). Se cita por primera vez para Puebla.

Material estudiado: MÉXICO. Puebla, municipio Honey, Cascadas Arcoíris, Rincón de Chila, 20¹5'32.4"N, 98¹4'48.3"O, 25.XI.2017, M. Sánchez 1079 (ENCB).

Notas taxonómicas: esta especie se caracteriza por apotecios de $45 \mathrm{~mm}$ de diámetro, cupuliformes-estipitados; ascas 240-300 × 14-18 $\mu \mathrm{m}$, cilíndricas; ascosporas 16.5-19.5 $\times 11-13 \mu \mathrm{m}$, elípticas, lisas, hialinas.

\section{Pezizaceae}

**Adelphella babingtonii (Berk. \& Broome) Pfister, Matočec \& I. Kušan, Mycologia Montenegrina 11: 14. 2009. Figs. 5C-F.

इ Peziza babingtonii Berk. \& Broome, Ann. Mag. nat. Hist., Ser. 2 7: 179. 1851. TIPO: GRAN BRETAÑA. Sin localidad, sobre madera podrida, s.f., Berkeley 554 (holotipo: Herb. Berkeley).

इ Psilopezia babingtonii (Berk. \& Broome) Berk., Outl.Brit. Fung. (London): 373. 1860.

三 Rhizina babingtonii (Berk. \& Broome) Massee \& Crossl., in Massee, Brit. Fung.-FI. (London) 4: 455. 1895.

इ Pachyella babingtonii (Berk. \& Broome) Boud., Hist. Class Discom. Eur. (Paris): 51. 1907.

इChaetothyrium babingtonii (Berk. \& Broome) Keissl., Robenhorst's Kryptogamen-Flora, Pyrenulaceae bis Mycoporaceae, Coniocarpineae 9(1, 2): 446. 1937

三 Adelphella babingtonii (Berk. \& Broome) Pfister, Matočec \&I. Kušan, Mycologia Montenegrina 10: 206. 2008.

Apotecio 5-20 × 1-2 mm, discoide, textura gelatinosa, color marrón amarillento (30C6) a marrón opaco (29E4), gregario o solitario; excípulo ectal 127-129.2 $\mu \mathrm{m}$ de grosor, textura globular a angularis, células 41.8-57× 26.6-47.5 $\mu \mathrm{m}$, globosas a angulares color marrón (30F7); excípulo medu- 
lar 104.5-731.5 $\mu \mathrm{m}$ de grosor, adelgazando hacia el margen con textura intrincada formada por hifas hialinas; himenio 354.2-385 $\mu \mathrm{m}$; paráfisis 8-12 $\mu \mathrm{m}$ de diámetro, filiformes con ápices clavados, hialinas; ascas 250-325 × 15-20 $\mu \mathrm{m}$, cilíndricas, octosporadas, uniseriadas, hialinas, inamiloides; ascosporas 17.6-20 × 10.4-12.8 $\mu \mathrm{m}$, oblongas a elípticas, hialinas, generalmente con dos gútulas, pared gruesa y lisa.

Hábito: saprobio, lignícola, creciendo sobre Quercus sp.

Distribución: Gran Bretaña (Berkeley y Broome, 1851); Estados Unidos de América (Beug et al., 2014), España (Requejo y Castro, 2015). Se cita por primera vez para México.

Material estudiado: MÉXICO. Puebla, municipio Honey, Cascadas Arcoíris, 20¹5'32.4"N, 98¹4'48.3"O, 25.XI.2017, M. Sánchez 1092 (ENCB), 1097 (ENCB).

Notas taxonómicas: la especie se caracteriza por presentar ascas de 250-325 × 15-20 $\mu \mathrm{m}$ y ascosporas de 17.6$20 \times 10.4-12.8 \mu \mathrm{m}$, oblongas a elípticas, hialinas. Pfister et al. (2008) la segregaron del género Pachyella Boud. por caracteres histoquímicos y citológicos.

\section{Pyronemataceae}

*Anthracobia melaloma (Alb. \& Schwein.) Arnould, Bull. Soc. Mycol. Fr. 9(2): 112. 1893.

三 Peziza melaloma Alb. \& Schwein., Cosnp. Fung. (Leipzig): 336. 1805. TIPO: FRANCIA. Sin localidad, sobre suelo quemado, s.f., Albertini y Schweinitz 1002 (tipo no localizado). ミPyronema melaloma (Alb. \& Schwein.) Fuckel, (as melalomum), Jb. Nassau. Ver. Naturk. 23-24: 319. 1870. 三 Aleuria melaloma (Alb. \& Schwein.) Gillet, Champignons de France, Discom. (2): 54. 1879.

三 Humaria melaloma (Alb. \& Schwein.) P. Karst., Acta Soc. Fauna Flora Fenn. 2(6): 120. 1885.

三 Lachnea melaloma (Alb. \& Schwein) Sacc., Syll. Fung. (Abellini) 8: 181. 1889.

三 Scutellinia melaloma (Alb. \& Schewein.) Kuntze, Revis. Gen. PI. (Leipzig) 2: 869. 1891.
ミ Humariella melaloma (Alb. \& Schwein.) J. Schröt., Krypt.FI. Schlesien (Breslau) 3.2(1-2): 37. 1893.

三 Patella melaloma (Alb. \& Schwein.) Seaver, North American Cup-Fungi, (Operculates) (New York): 167. 1928.

Hábito: saprobio, pirófilo.

Distribución: Oaxaca (Raymundo et al., 2013). Se cita por primera vez para Puebla.

Material estudiado: MÉXICO. Puebla, municipio Honey, camino a la Cascada Velo de Novia, Chila de Juárez, 20¹7'22.41'N, 98¹3'69.67'O, 21.X.2017, M. Sánchez 1051 (ENCB).

Notas taxonómicas: se caracteriza por apotecios discoides, color naranja; ascas 85-144 × 8-9 $\mu \mathrm{m}$, cilíndricas; ascosporas 13.3-15.2 × 5.7-7.6 $\mu \mathrm{m}$ elipsoides, hialinas.

*Scutellinia pennsylvanica (Seaver) Denison, Mycologia 51(5): 619. 1961.

三 Melastiza pennsylvanica Seaver, North American Cup-Fungi, (Opreculates) (New York): 104. 1928. TIPO: ESTADOS UNIDOS DE AMÉRICA. Pennsylvania, Youghiogheny River, sobre madera podrida, s.f., Seaver s.n. (no se menciona en cual herbario se depositó el espécimen tipo).

三 Melastiziella pennsylvanica (Seaver) Svrček, Acta Mus. Nat. Prag. 4B(6): 61. 1948.

Hábito: saprobio, lignícola, sobre madera en descomposición.

Distribución: Chihuahua, Estado de México, Guanajuato, Guerrero, Michoacán, Querétaro y Tlaxcala (Sierra et al., 2016). Se cita por primera vez para Puebla.

Material estudiado: MÉXICO. Puebla, municipio Honey, camino a la Cascada Velo de Novia, Chila de Juárez, 20¹7'22.41'N, 98¹3'69.67'O, 21.X.2017, M. Sánchez 1071 (ENCB), 1073 (ENCB); Cascadas Arcoíris, Rincón de Chila, $20^{\circ} 15^{\prime} 32.4^{\prime \prime} \mathrm{N}, 98^{\circ} 14^{\prime} 48.3^{\prime \prime O}$, 25.XI.2017, M. Sánchez 1083 (ENCB). 
Notas taxonómicas: se caracteriza por apotecios discoides, color amarillo a rojo-naranja, con setas al margen; ascas 140-220 × 10-14 $\mu \mathrm{m}$, cilíndricas; ascosporas 16-19 $\times 10.5-13 \mu \mathrm{m}$, elipsoides, hialinas, verrugas redondeadas uniéndose, formando crestas.

*Scutellinia scutellata (L.) Lambotte, Mém. Soc. Roy. Sci., Liége, Série 2 14: 299. 1887.

TIPO: SUECIA. Sin localidad, sobre madera podrida, s.f., Linnaeus s.n. (tipo no localizado).

三 Peziza scutellata L., Sp. PI. 2: 1181. 1753.

三 Peziza aurantica Bull., Hist. Champ. Fr. (Paris) 2(2): 685. 1792.

इ Lachnea scutellata (L.) Gillet, Champignons de France, Discom. (3): 57. 1880.

इ Humariella scutellata (L.) J. Schröt., Krypt.-FI. (Breslau) 3.2(1-2): 37. 1893.

इ Patella scutelata (L.) Morgan, J. Mycol. 8(4): 187. 1902.

三 Ciliaria scutellata (L.) Quél. ex Boud., Hist. Class. Discom. Eur. (Paris): 61. 1907.

Hábito: saprobio, crece sobre madera en descomposición.

Distribución: Estado de México, Guanajuato, Guerrero, Michoacán, Nayarit y Tlaxcala (Sierra et al., 2016), Hidalgo (Frutis y Guzmán, 1983); Sonora (Pérez-Silva et al., 1996). Se cita por primera vez para Puebla.

Material estudiado: MÉXICO. Puebla, municipio Honey, Parque Turístico El Sabinal, $20^{\circ} 14^{\prime} 05.1^{\prime \prime} \mathrm{N}, 98^{\circ} 12^{\prime} 49.8^{\prime \prime O}$, 17.XI.2016, M. Sánchez 755 (ENCB, FEZA); Cascadas Arcoíris, Rincón de Chila, 20¹5'32.4"N, 98¹4'48.3'O, 25.XI.2017, M. Sánchez 1100 (ENCB).

Notas taxonómicas: se caracteriza por apotecios discoides, color naranja pálido a naranja rojizo, con setas al margen; ascas 270-285 × 16-18 $\mu \mathrm{m}$, cilíndricas; ascosporas 17-21 (22) × (9) 10-13 $\mu \mathrm{m}$, elipsoides, hialinas, ornamentadas con verrugas irregulares.

\section{Sarcoscyphaceae}

*Cookeina venezuelae (Berk. \& M. A. Curtis) Le Gal, Discomyc. Madagascar (Paris): 239. 1953.

इ Peziza venezuelae Berk. \& M.A. Curtis, Mycogr. Discom. (London) 1(3): 120. 1876. TIPO: VENEZUELA. Sin localidad, sobre el suelo, s.f., M. J. Berkeley y M. A. Curtis s.n. (holotipo: Herb. Berkeley).

三 Phillipsia venezuelae (Berk. \& M.A. Curtis ex Cooke) Massee, J. Linn. Soc., Bot. 31: 473. 1896.

Hábito: saprobio, lignícola en madera en descomposición.

Distribución: Jalisco (Herrera et al., 2002); Nayarit, Oaxaca, San Luis Potosí y Tamaulipas (Ortega-López et al., 2019). Se cita por primera vez para Puebla.

Material estudiado: MÉXICO. Puebla, municipio Honey, riachuelo cerca del Río San Marcos, San Pedro Chila, 20¹7'38.78' 'N, 98¹3'24.69"O, 17.IX.2017, M. Sánchez 946 (ENCB), 952 (ENCB); camino a la Cascada Velo de Novia, Chila de Juárez, 20¹7'22.41'N, 98¹3'69.67"O, 21.X.2017, M. Sánchez 1054 (ENCB).

Notas taxonómicas: se caracteriza por apotecios en forma de copa, color naranja-rosado; ascas 275-350 × 10$14 \mu \mathrm{m}$, suboperculadas; ascosporas (30)32-40 × 11.4-14 $\mu \mathrm{m}$, elipsoides a fusoides, hialinas, con estrías longitudinales conectadas entre ellas con estrías más finas.

*Phillipsia olivacea Rick, Brotéria, sér. Bot. 25(2-3): 86. 1931.

TIPO: BRASIL. Sin localidad, sobre el suelo, s.f., Rick s.n. (tipo no localizado).

Hábito: saprobio, lignícola.

Distribución: San Luis Potosí (Ortega-López et al., 2019). Se cita por primera vez para Puebla. 
Material estudiado: MÉXICO. Puebla, municipio Honey, riachuelo cerca del Río San Marcos, San Pedro Chila, $20^{\circ} 17^{\prime} 38.78^{\prime \prime} \mathrm{N}, 98^{\circ} 13^{\prime 24.69 " O, ~ 17 . I X .2017, ~ M . ~ S a ́ n c h e z ~}$ 948 (ENCB).

Notas taxonómicas: se caracteriza por apotecios cupuliformes a discoides, color amarillo-naranja, consistencia gelatinosa; ascas 350-470 × 14-18 $\mu \mathrm{m}$, cilíndricas, pediceladas; ascosporas 21-28.5 × (7.6)9.5-11.4 $\mu \mathrm{m}$, elipsoides, hialinas, rugosas de forma irregular en toda la espora.

\section{Sarcosomataceae}

*Plectania rhytidia (Berk.) Nannf. \& Korf, Mycologia 49(1): 110. 1957.

三 Peziza rhytidia Berk., Bot. Antarct. Voy. Erebus Terror 1839-1843, II, FI. Nov.-Zeal.: 200. 1855. TIPO: NUEVA ZELANDA. Bahía de las Islas, sobre el suelo, s.f., Berkeley s.n. (holotipo: K).

इUrnula rhytidia (Berk.) Cooke, Syll. Fung. (Abellini) 8: 548. 1889.

इ Sarcosoma rhytidium (Berk.) Le Gal (as "rhytidia), Discomyc. Madagascar (Paris): 224. 1953.

Hábito: saprobio, lignícola.

Distribución: Hidalgo (Raymundo et al., 2019); Veracruz (Medel y Chacón, 2000). Se cita por primera vez para Puebla.

Material estudiado: MÉXICO. Puebla, municipio Honey, Cascadas Arcoíris, Rincón de Chila, 20¹5'32.4"N, 98¹4'48.3"O, 25.XI.2017, M. Sánchez 1077 (ENCB).

Notas taxonómicas: esta especie se caracteriza por formar apotecios 5-20 mm de diámetro, color negro, cupuliformes, sésil o subestipitado; ascas 171-188 × 11-13.6 $\mu \mathrm{m}$, cilíndricas; ascosporas 18-24 × 9.5-12 $\mu \mathrm{m}$, elipsoidales, hialinas, con estrías transversales anastomosadas.

*Pseudoplectania nigrella (Pers.) Fuckel, Jb. Nassau. Ver. Naturk. 23-24: 324. 1870.
TIPO: AUSTRIA. En hojas de pino podrido en bosques de abeto, s.f., Persoon s.n. (tipo no localizado).

इ Helvella hemisphaerica Wulfen, Schr. Ges. Natuf. Freunde, Berlin 8: 141. 1788.

इ Peziza nigrella Pers., Syn. Meth. Fung. (Göttingen) 2: 648. 1801.

इ Lachnea nigrella (Pers.) Gillet, Champignons de France, Discom. (3): 78. 1880.

इ Plectania nigrella (Pers.) P. Karst., Acta Soc. Fauna Flora Fenn. 2(no. 6): 119. 1885.

三 Crouania nigrella (Pers.) Hazsl., Mathem. Természettud. Közlem. Magg. Tudom. Akad. 21: 261. 1886.

इScypharia nigrella (Pers.) Quél., Enchir. Fung. (Paris): 283. 1886.

三Sepultaria nigrella (Pers.) Lambotte, Mém. Soc. Roy. Sci. Liège, Série 2 14: 301.1887.

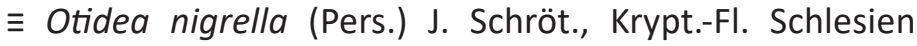
(Breslau) 3.2(1-2): 48. 1893.

इSphaerospora nigrella (Pers.) Massee, Brit. Fung-Fl. (London) 4: 296. 1895.

Hábito: saprobio, lignícola.

Distribución: Hidalgo (Raymundo et al., 2016; 2019); Estado de México, Guerrero (Pompa-González y Cifuentes, 1991); Veracruz (Medel et al., 2006). Se cita por primera vez para Puebla.

Material estudiado: MÉXICO. Puebla, municipio Honey, camino a la Cascada Velo de Novia, Chila de Juárez, 20¹7'22.41"N, 98¹3'69.67"O, 21.X.2017, M. Sánchez 1048 (ENCB); Cascadas Arcoíris, Rincón de Chila, $20^{\circ} 15^{\prime} 32.4^{\prime \prime} \mathrm{N}, 98^{\circ} 14^{\prime} 48.3^{\prime \prime} \mathrm{O}, 25 . X I .2017$, M. Sánchez 1080 (ENCB).

Notas taxonómicas: se caracteriza por apotecios discoides a cupuliformes, color marrón oscuro a negro; ascas; 250-320 × 12-17 $\mu \mathrm{m}$, cilíndricas; ascosporas 10-12 $\mu \mathrm{m}$, globosas, lisas, hialinas.

*Urnula mexicana (Ellis \& Holw.) M. Carbone, Agnello. A.D. Parker \& P. Alvarado, Ascomycete.org 5(1): 14. 2012. 
इ Bulgaria mexicana Ellis \& Holw., Bot. Gaz. 24(1): 37. 1897. TIPO. MÉXICO. Morelos, Cuernavaca, IX.1986, E. W. D. Holway s.n. (holotipo: NY 01169254).

इ Sarcosoma mexicanum (Ellis \& Holw.) Paden \& Tylutki, Mycologia 61(3): 689. 1969.

इ Plectania mexicana (Ellis \& Holw.) Paden, Fl. Neotrop., Monogr. 37: 7. 1983.

Hábito: saprobio, creciendo sobre Quercus sp.

Distribución: Hidalgo (Frutis y Guzmán, 1983; Raymundo et al., 2016), Michoacán (Díaz-Barriga et al., 1988), Morelos (Paden, 1983), Tamaulipas (Heredia, 1989) y Veracruz (Medel y Chacón, 2000). Se cita por primera vez para Puebla.

Material estudiado: MÉXICO. Puebla, municipio Honey, Cascadas Arcoíris, Rincón de Chila, 20¹5'32.4"N, 98¹4'48.3"O, 25.XI.2017, M. Sánchez 1087 (ENCB).

Notas taxonómicas: se caracteriza por apotecios de 14-35 mm de diámetro, cupuliformes, carnoso-gelatinosos, color oscuro; ascas 250-270 × 13-15(16) $\mu \mathrm{m}$, cilíndricas; ascosporas 25-34 × 8-10 $\mu \mathrm{m}$, alantoides, subhialinas.

\section{Sordariomycetes}

Hypocreales

\section{Hypocreaceae}

*Hypomyces chrysospermus Tul \& C. Tul., Annls Sci. Nat., Bot., Sér. 4 (13): 16. 1860.

TIPO: NUEVA ZELANDA. Bahía de las Islas, sobre boletos, s.f., L. R. Tulasne y C. Tulasne s.n. (espécimen tipo no designado, ni se menciona en cual herbario se depositó).

इ Reticularia chrysosperma Bull., Herb. Fr. (Paris) 10: tab. 476. 1790.

इ Mucor chrysospermus (Bull.) Bull., Hist. Champ. Fr. (Paris) 1(1): 99. 1791.

इ Mycobanche chrysosperma (Bull.) Pers., Traité Champ. Comest. (Paris): 133. 1818.
इSepedonium chrysospermum (Bull.) Fr., Syst. Mycol. (Lundae) 3(2): 438. 1832.

इ Hypolyssus chrysospermus (Tul. \& C. Tul.) Kuntze, Revis. Gen. Pl. (Leipzig) 3(3): 488. 1898.

$\equiv$ Apiocrea chrysosperma (Tul. \& C. Tul.) Syd. \& P. Syd., Annls. Mycol. 18(4/6): 187. 1921.

Hábito: epífito, fungícola sobre distintas especies de Boletales.

Distribución: Jalisco (Rodríguez-Alcántar et al., 2018; Gándara et al., 2014). Se cita por primera vez para Puebla.

Material estudiado: MÉXICO. Puebla, municipio Honey, Parque Turístico El Sabinal, 20¹4'05.1"N, $98^{\circ} 12^{\prime} 49.8^{\prime \prime O}$, 17.XI.2016, M. Sánchez 770 (ENCB, FEZA).

Notas taxonómicas: esta especie se caracteriza por parasitar distintas especies de Boletales, color amarillo; ascas 100-140 × 5-8 $\mu \mathrm{m}$, cilíndricas; ascosporas 20-25 × 4-5 $\mu \mathrm{m}$, fusiformes, hialinas, con un septo.

Hypomyces hyalinus Tul \& C. Tul., Annls Sci. Nat., Bot., sér. 4(13): 11.1860.

三Sphaeria hyalina Schwein., Schr. Naturf. Ges. Leipzig 1: 30. 1822. TIPO: Sin localidad, sobre Amanita, s.f., Schweinitz s.n. (tipo no localizado).

इ Hypocrea hyalina (Schwein.) Fr., Summa Veg. Scand., Sectio Post (Stockholm): 383. 1849.

इ Peckiella hyalina (Schwein.) Sacc., Syll. Fung. (Abellini) 9: 945. 1891.

इ Hypolyssus hyalinus (Schwein.) Kuntze, Revis. Gen. PI. (Leipzig) 3(3): 488. 1898.

$\equiv$ Apiocrea hyalina (Schwein.) Syd. \& P. Syd., Annls. Mycol. 18(4/6): 187. 1921.

Hábito: epífito, fungícola sobre Amanita rubescens Pers.

Distribución: Jalisco (Gándara et al., 2014); Hidalgo (Frutis y Guzmán, 1983); Puebla (Martínez-Alfaro et al., 1983). 
Material estudiado: MÉXICO. Puebla, municipio Honey, camino a la Cascada Velo de Novia, Chila de Juárez,

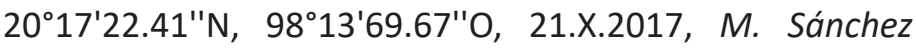
1066 (ENCB).

Notas taxonómicas: esta especie se caracteriza por peritecios de $250-325 \times 180-210 \mu \mathrm{m}$, ovoides en los basidiomas de la especie que parasita; ascas 110-130 × 4-6 $\mu \mathrm{m}$, cilíndricas; ascosporas 15-20 × 4.5-6.5 $\mu \mathrm{m}$, fusiformes, hialinas, con un septo.

\section{Nectriaceae}

*Cosmospora ustulinae (Teng) C.S. Herrera \& P. Chaverri, Mycologia 107(3): 550. 2015.

E Nectria ustulinae Teng, Sinensia, Shangai 4: 275. 1934. TIPO: CHINA. Shangai, sobre estroma de Ustulina vulgaris, s.f., Teng s.n. (espécimen tipo no designado, ni se menciona en cual herbario se depositó).

三 Dialonectria ustulinae (Teng) Lar. N. Vassiljeva, Nizshie Rasteniya, Griby i Mokhoobraznye Dalnego Vostoka Rossi (Sankt-Peterburg): 171. 1998.

Hábito: epífito, fungícola crece sobre Kretzschmaria deusta (Hoffm.) P.M.D. Martin.

Distribución: Hidalgo (Raymundo et al., 2016; 2019). Se cita por primera vez para Puebla.

Material estudiado: MÉXICO. Puebla, municipio Honey, riachuelo cerca del Río San Marcos, San Pedro Chila, 20¹7'38.78"N, 98¹3'24.69"O, 17.IX.2017, M. Sánchez 956 (ENCB).

Notas taxonómicas: se caracteriza por peritecios solitarios a gregarios, subglobosos, color rojo; ascas 41-61 $\times$ 4-5 $\mu \mathrm{m}$, clavadas; ascosporas 6-8 × 2.5-4.5 $\mu \mathrm{m}$, elipsoidales, verrugosas, con un septo, hialinas.

*Thelonectria ostrina Salgado \& P. Chaverri, Fungal Diversity 70: 1-29. 2015.
TIPO: PUERTO RICO. Río Grande, Montañas Liquillo, Bosque Nacional del Caribe, sobre corteza de árbol no identificado, 24.II.1996, G. J. Samuels et al. 8097 (holotipo: BPI 745542).

Hábito: saprobio, lignícola.

Distribución: Hidalgo (Raymundo et al., 2017). Se cita por primera vez para Puebla.

Material estudiado: MÉXICO. Puebla, municipio Honey, Cascadas Arcoíris, Rincón de Chila, 20¹5'32.4"N, 98 14'48.3"O, 26.Il.2018, T. Raymundo 7565 (ENCB); loc. cit., 26.II.2018, M. Sánchez 1160-A (ENCB); loc. cit., 26.II.2018, R. Valenzuela 18038 (ENCB).

Notas taxonómicas: se caracteriza por peritecios de 300-600 × 250-400 $\mu \mathrm{m}$, solitarios a gregarios, globosos, color rojo intenso, cambiando a carmín en $\mathrm{KOH}$; ascas 64-96 × 8.8-9.6 $\mu \mathrm{m}$, cilíndricas a clavadas; ascosporas 9.6-12 × 4.4$5.6 \mu \mathrm{m}$, uniseriadas, con un septo, elipsoidales, hialinas.

*Thelonectria veuillotiana (Roum. \& Sacc.) P. Chaverri \& Salgado, Stud. Mycol. 68: 77. 2011.

इ Dialonectria veuillotiana (Roum. \& Sacc.) Cooke, Grevillea 12(64): 110. 1884.

इ Cucurbitaria veuillotiana (Roum. \& Sacc.) Kuntze, Revis. Gen. PI. (Leipzig) 3(3): 462. 1898.

इ Neonectria veuillotiana (Roum. \& Sacc.) Mantiri \& Samuels, Can. J. Bot. 79(3): 339. 2001. TIPO: FRANCIA. Lyon, sobre corteza podrida, 1879, Saccardo y Roumeguere s.n. (espécimen tipo no designado, ni se menciona en cual herbario se depositó).

Hábito: saprobio, lignícola.

Distribución: Hidalgo (Raymundo et al., 2017; 2019). Se cita por primera vez para Puebla.

Material estudiado: MÉXICO. Puebla, municipio Honey, Cascadas Arcoíris, Rincón de Chila, 20¹5'32.4"N, 98¹4'48.3"O, 25.XI.2017, M. Sánchez 1109 (ENCB). 
Notas taxonómicas: esta especie se caracteriza por formar peritecios de 280-400 × 250-350 $\mu \mathrm{m}$, solitarios a gregarios, subglobosos, color rojo anaranjado; ascas 66.5-98.8 $\times$ 6-9.5 $\mu \mathrm{m}$, hialinas; ascosporas 16-18.4 × 5-6.5 $\mu \mathrm{m}$, elipsoides, hialinas.

\section{Ophiocordycipitaceae}

Tolypocladium capitatum (Holmsk.) C.A. Quandt, Kepler \& Spatafora, IMA Fungus 5(1): 126. 2014.

三 Clavaria capitata Holmsk., Beata Ruris Otia Fungis Danicis 1: 38. 1790. TIPO: SUECIA. Kinnekulle, ocasionalmente en pino, s.f., Holmskjold s.n. (espécimen tipo no designado, ni se menciona en cual herbario se depositó).

इ Sphaeria capitata (Holmsk.) Pers., Comm Fung. Clav. (Lipsiae): 13. 1797.

इCordyceps capitata (Holmsk.) Fr., Observ. Mycol. (Havniae) 2: 317. 1818.

$\equiv$ Torrubia capitata (Holmsk.) Tul \& C. Tul., Select. Fung. Carpol. (Paris) 3: 22. 1865.

三Elaphocordyceps caitata (Holmsk.) G.H. Sung, J.M. Sung \& Spatafora, Stud. Mycol. 57: 37. 2007.

Hábito: epífito, fungícola crece sobre Elaphomyces sp.

Distribución: Puebla (Martínez-Alfaro et al., 1983); Hidalgo (Frutis y Guzmán, 1983; Raymundo et al., 2019); Sonora (Pérez-Silva et al., 1996).

Material estudiado: MÉXICO. Puebla, municipio Honey, camino a la Cascada Velo de Novia, Chila de Juárez, $20^{\circ} 17^{\prime} 22.41^{\prime \prime} \mathrm{N}, 98^{\circ} 13^{\prime} 69.67^{\prime \prime O}, 21 . X .2017$, M. Sánchez 1058 (ENCB).

Notas taxonómicas: citada anteriormente como Cordyceps capitata (Holmsk.) Fr., se caracteriza por presentar ascas 350-540 × 10-12 $\mu \mathrm{m}$, cilíndricas; ascosporas 8-25 $\times 1.5-3 \mu \mathrm{m}$, cilíndricas, multiseptadas, hialinas.

Magnaporthales

Ophioceraceae
**Ophioceras leptosporum (S.H. Iqbal) J. Walker, Mycotaxon 11(1): 62. 1980. Fig. 6.

इ Gaeumannomyces leptosporus S.H. Iqbal, Trans. Br. Mycol. Soc. 58(2): 346. 1972. TIPO: GRAN BRETAÑA. Exeter, cerca del río Creedy, en tallos podridos de Umbelliferae, 6.II.1970, S. H. Iqbal s.n. (holotipo: Herbarium Mycologicum Universitas Exeter).

Peritecios 470-716 × 346-508 $\mu \mathrm{m}$ de diámetro, solitarios a gregarios, superficiales, subglobosos a piriformes, con un cuello alargado 2618-3465 × 72-102 $\mu \mathrm{m}$, color negro, ostiolo más claro que el resto del ascoma, textura carbonosa, las paredes del peritecio son de una textura angular; perífisis y paráfisis ausentes; ascas 80-100 × 5.5-6.5 $\mu \mathrm{m}$, hialinas cilíndricas, rectas a ligeramente curvas, octosporadas, biseriadas, inamiloides; ascosporas (48.8)58.4$68.8 \times 1.6 \mu \mathrm{m}$, filiformes, rectas o sigmoides, hialinas, pared delgada y lisa, multiseptadas.

Hábito: saprobio, lignícola, creciendo sobre madera en descomposición.

Distribución: Gran Bretaña (Iqbal, 1972); Estados Unidos de América (Shearer et al., 1999). Se cita por primera vez para México.

Material estudiado: MÉXICO. Puebla, municipio Honey, San Pedro Chila, 20¹7'38.78"N, 98¹3'24.69"O, 17.IX.2017, M. Sánchez 950 (ENCB).

Notas taxonómicas: se caracteriza por la ausencia de perífisis y paráfisis; ascas 80-100 × 5.5-6.5 $\mu \mathrm{m}$, cilíndricas, rectas a ligeramente curvas; ascosporas (48.8)58.4-68.8 $\times$ $1.6 \mu \mathrm{m}$. La única especie del género que había sido citada en México es $O$. commune Shearer, J.L. Crane \& W. Chen, como un ascomiceto dulce acuícola de la Ciudad de México (Chavarria et al., 2010).

Xylariales

Hypoxylaceae 

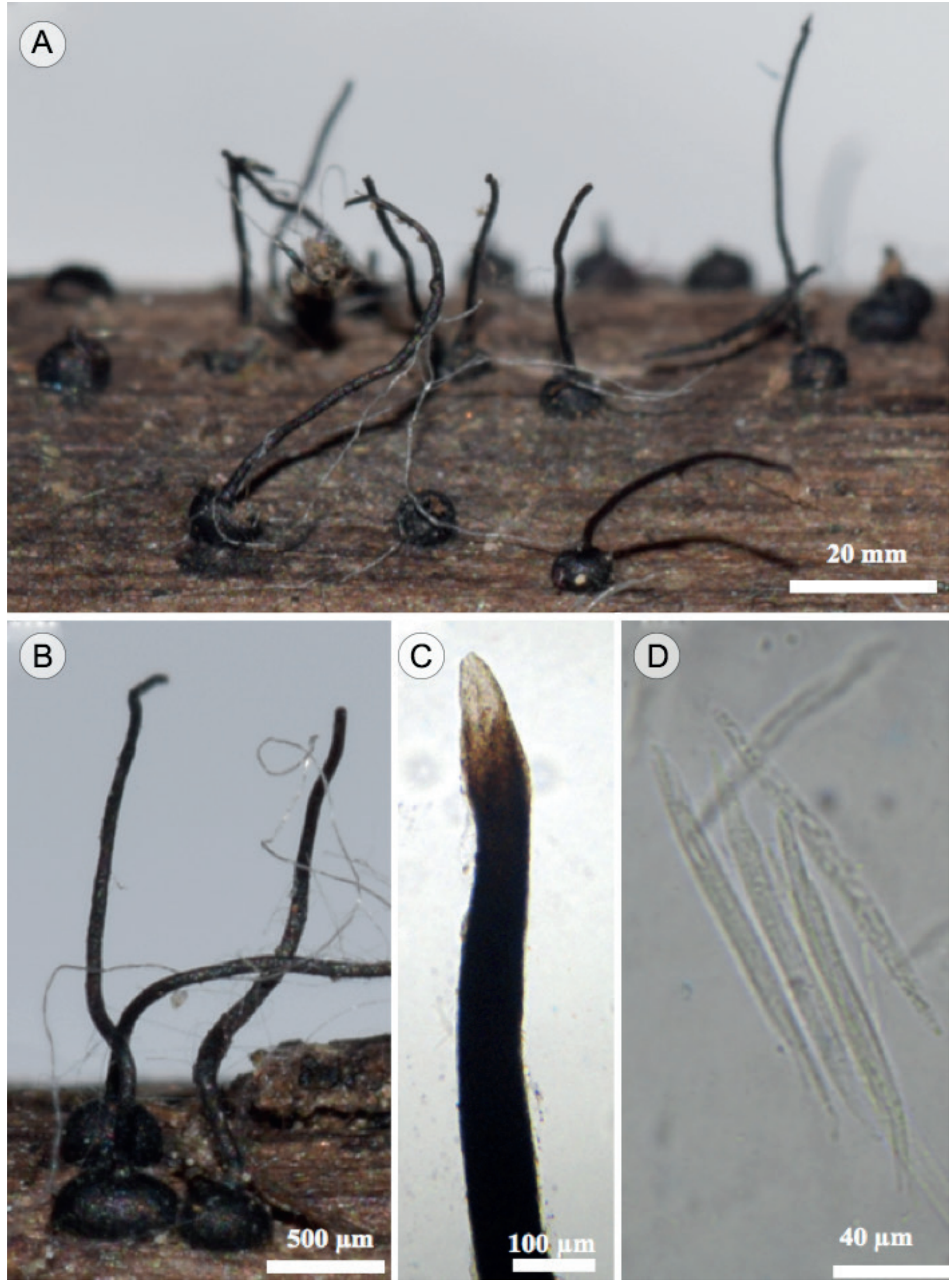

D

Figura 6: Ophioceras leptosporum (S.H. Iqbal) J. Walker: A-B. peritecios; C. cuello del peritecio; D. ascas con ascosporas. 
*Annulohypoxylon annulatum (Schwein.) Y.M. Ju, J.D. Rogers \& H.M. Hsieh, Mycologia 97(4): 857. 2005.

इ Sphaeria annulata Schwein., J. Acad. Nat. Sci. Philad. 5: 11. 1825. TIPO: ESTADOS UNIDOS DE AMÉRICA. Philadelphia, s.f., Sweinitz s.n. (lectotipo: PH 01106655).

三 Hypoxylon annulatum (Schwein.) Mont., Hist. Fis. y Polit. Chile, Bot. 7: 445. 1850.

Hábito: endófito, lignícola, creciendo en Quercus sp.

Distribución: Chiapas y Tamaulipas (San Martín et al., 1999b). Se cita por primera vez para Puebla.

Material estudiado: MÉXICO. Puebla, municipio Honey, Río de Honey, 20¹4'38"N, 98¹2'35.4"O, 05.V.2016, M. Sánchez 405 (ENCB, FEZA); Cascadas Arcoíris, Rincón de Chila, $20^{\circ} 15^{\prime} 32.4^{\prime \prime N}, 98^{\circ} 14^{\prime} 48.3^{\prime \prime O}$, 25.XI.2017, M. Sánchez 1091 (ENCB).

Notas taxonómicas: esta especie se caracteriza por estromas hemisféricos, color negro con pigmentos de color verde oliváceo en contacto con $\mathrm{KOH}$ al $10 \%$; ascas $70-80$ $\times$ 5-6 $\mu \mathrm{m}$, cilíndricas, poro apical amiloide; ascosporas 7.5$10.5 \times 3.5-5 \mu \mathrm{m}$, elipsoidales, color marrón.

Annulohypoxylon thouarsianum (Lév.) Y.M. Ju, J.D. Rogers \& H.M. Hsieh, Mycologia 97(4): 861. 2005.

इ Sphaeria thouarsiana Lév., Annls. Sci. Nat., Bot., Sér. 3(5): 258. 1846. TIPO: ECUADOR. Islas Galápagos, sobre madera, s.f., Lévillé s.n. (holotipo: herb. Museum Paris).

三 Daldinia thouarsiana (Lév.) Sacc., Syll. Fung. (Abellini) 1: 395. 1882.

三 Hemisphaeria thouarsiana (Lév.) Kuntze, Revis. Gen. PI. (Leipzig) 3(3): 482. 1898.

इ Hypoxylon thouarsianum (Lév.) Lloyd, Mycol. Writ. 5: 26. 1919.

Hábito: endófito, lignícola, creciendo en Quercus sp.

Distribución: Durango (Raymundo et al., 2012); Hidalgo (Frutis y Guzmán, 1983; Raymundo et al., 2016;2019);
Nuevo León (Chacón y Guzmán, 1983); Oaxaca (Raymundo et al., 2013); Puebla (Martínez-Alfaro et al., 1983).

Material estudiado: MÉXICO. Puebla, municipio Honey, Río de Honey, 20¹4'38"N, 98¹2'35.4"O, 05.V.2016, M. Sánchez 412 (ENCB, FEZA), 414 (ENCB, FEZA), 415 (ENCB, FEZA); Parque Turístico El Sabinal, 20¹4'05.1'N, 98¹2'49.8"O, 17.XI.2016, M. Sánchez 765-A (ENCB, FEZA); Cascadas Arcoíris, Rincón de Chila, 20¹5'32.4"N, 98 $14^{\prime} 48.3^{\prime \prime O}$, 25.XI.2017, S. Rangel 2 (ENCB), 3 (ENCB); loc. cit., 26.II.2018, U. Rodríguez y D. Jiménez 116 (ENCB); loc. cit., 26.II.2018, M. Sánchez 1082 (ENCB), 1085 (ENCB), 1096 (ENCB), 1099 (ENCB).

Notas taxonómicas: se caracteriza por estromas hemisféricos, color negro con pigmentos de color verde oliva en contacto con $\mathrm{KOH}$ al 10\%; ascas 130-140 × 6-10 $\mu \mathrm{m}$, cilíndricas, poro apical amiloide; ascosporas 14-24 × 4.5-5 $\mu \mathrm{m}$, elipsoidales, color marrón.

*Hypoxylon perforatum (Schwein.) Fr., Summa veg. Scand., Sectio Post. (Stockholm): 384. 1849.

इ Sphaeria perforata Schwein., Schr. naturf. Ges. Leipzig 1: 31. 1822. TIPO: ESTADOS UNIDOS DE AMÉRICA. Pennsylvania, Northampton County, Bethlehem, sobre corteza, s.f., L.D. Schweinitz 1194 (lectotipo: PH); Carolina del Norte, Salem, sobre corteza, s.f., Collins 28 (isolectotipos: BPI, PH). इ Hypoxylon rubiginosum var. perforatum (Schwein.) L.E. Petrini, Mycol. Helv. 1(7): 531. 1986.

Hábito: endófito, lignícola, sobre madera de Quercus sp.

Distribución: Nuevo León (San Martín et al., 1999a). Se cita por primera vez para Puebla.

Material estudiado: MÉXICO. Puebla, municipio Honey, Cascadas Arcoíris, Rincón de Chila, 20¹5'32.4"N, 98¹4'48.3"O, 26.II.2018, M. Sánchez 1149 (ENCB).

Notas taxonómicas: esta especie se caracteriza por estromas resupinados, color marrón rojizo, con pigmentos de color ámbar al contacto con $\mathrm{KOH}$ al $10 \%$; ascas $60-80 \times$ 
6-8 $\mu \mathrm{m}$, cilíndricas, poro apical amiloide; ascosporas 9-12 $\times$ 4-6 $\mu \mathrm{m}$, color marrón.

\section{Graphostromataceae}

*Biscogniauxia nummularia (Bull.) Kuntze, Rev. gen. pl. (Leipzig) 2: 398. 1891.

इ Hypoxylon nummularium Bull., Herb. Fr. (Paris): tab. 468. 1790. TIPO: FRANCIA. En ramas de diferentes árboles, s.f., Bulliardii s.n. (lectotipo: Bulliardii, Histoire des champignons de la France).

三 Sphaeria nummularia DC., Fl. Franç., Edn 3 (Paris) 2: 290. 1805.

= Nummularia nummularia (Bull.) J. Schröt., Krypt.-Fl. Schlesien (Breslau) 3.2(4): 458. 1897.

$=$ Nummularia nummularia (Bull.) House, N.Y. St. Mus. Bull. 266: 49. 1925.

Hábito: patógeno, lignícola, crece sobre Quercus sp.

Distribución: Hidalgo (Raymundo et al., 2016; 2019); Tamaulipas (Ju et al., 1998). Se cita por primera vez para Puebla.

Material estudiado: MÉXICO. Puebla, municipio Honey, Cascadas Arcoíris, Rincón de Chila, 20¹5'32.4"N, 98¹4'48.3'O, 26.II.2018, M. Sánchez 1147 (ENCB).

Notas taxonómicas: se caracteriza por estromas aplanados, color negro, carbonáceos; ascas 70-90 × 8-10 $\mu \mathrm{m}$, cilíndricas, poro apical amiloide; ascosporas 10-12 × 7.2-8.4 $\mu \mathrm{m}$, elipsoidales, línea germinal recta, color marrón.

\section{Xylariaceae}

*Kretzschmaria deusta (Hoffm.) P.M.D. Martin, South African Journal of Botany 36(2): 80. 1970.

इ Sphaeria deusta Hoffm., Vegetabilia Cryptogama 1: 3. 1787. TIPO: SUDÁFRICA. Sin localidad, sobre las raíces y troncos podridos de árboles, s.f., Hoffman s.n. (tipo no localizado).
Hábito: patógeno, lignícola crece sobre Quercus sp.

Distribución: Hidalgo (Raymundo et al., 2016; 2019); Oaxaca (Raymundo et al., 2014); Tamaulipas (San Martín y Lavín, 1997). Se cita por primera vez para Puebla.

Material estudiado: MÉXICO. Puebla, municipio Honey, riachuelo cerca del Río San Marcos, San Pedro Chila, 20¹7'38.78"N, 98¹3'24.69"O, 17.IX.2017, M. Sánchez 957 (ENCB); Cascadas Arcoíris, Rincón de Chila, 20¹5'32.4"N, 98¹4'48.3"O, 26.II.2018, R. Valenzuela 18035 (ENCB).

Notas taxonómicas: esta especie se caracteriza por estromas aplanados, color negro, carbonáceos, forma irregular; ascas 410-480 × 12-15 $\mu \mathrm{m}$, cilíndricas; ascosporas 28-34 × 7-9.5 $\mu \mathrm{m}$, irregularmente elipsoidales, color marrón.

*Xylaria anisopleura (Mont.) Fr., Nova Acta R. Soc. Scient. Upsal., Ser. 3, 1(1): 127. 1851.

इ Hypoxylon anisopleuron Mont., Annls. Sci. Nat. Bot., Sér. 2, 13: 348. 1840. TIPO: GUYANA FRANCESA. Sin localidad, sobre madera podrida, s.f., Maio 1838 (tipo no localizado). 三Xylosphaera anisopleura (Mont.) Dennis, Kew Bull. 13(1): 102. 1958

Hábito: saprobio, lignícola, crece sobre madera de Quercus sp.

Distribución: Chiapas, Oaxaca, Quintana Roo, Tabasco, Tamaulipas, Veracruz (San Martín y Rogers, 1989; 1995); Hidalgo (Raymundo et al., 2016); Veracruz (PérezSilva, 1975). Se cita por primera vez para Puebla.

Material estudiado: MÉXICO. Puebla, municipio Honey, camino a la Cascada Velo de Novia, Chila de Juárez, $20^{\circ} 17^{\prime} 22.41^{\prime \prime N}$, 98¹3'69.67"O, 21.X.2017, M. Sánchez 1072 (ENCB).

Notas taxonómicas: se caracteriza por estromas fragiformes a hemisféricos, negros, textura dura a carbonosa; ascas 230-275 × 8-10 $\mu \mathrm{m}$, cilíndricas, poro apical amiloide; 
ascosporas 20.9-24.7 × 7.6-9.5 $\mu \mathrm{m}$, elipsoides, color marón, línea germinal oblicua.

*Xylaria apiculata Cooke, Grevillea 8(46): 66. 1879.

TIPO: NUEVA ZELANDA. Maungaroa, sobre madera, s.f., Cooke s.n. (holotipo: K).

ミXylosphaera apiculata (Cooke) Dennis, Kew Bull. 13(1): 102. 1958.

Hábito: saprobio, lignícola, crece sobre madera.

Distribución: Chiapas, Tamaulipas (San Martín y Rogers, 1995). Se cita por primera vez para Puebla.

Material estudiado: MÉXICO. Puebla, municipio Honey, Parque Turístico El Sabinal, 20¹4'05.1"N, 98¹2'49.8"O, 17.XI.2016, M. Sánchez 765-B (ENCB).

Notas taxonómicas: esta especie se caracteriza por formar estromas simples, cilíndricos, con el ápice estéril, color negro; ascas 229-280 × 7-9 $\mu \mathrm{m}$, cilíndricas, poro apical amiloide; ascosporas 20-28 × 7-12 $\mu \mathrm{m}$, elipsoidales, color marón, línea germinal recta.

*Xylaria arbuscula Sacc., Michelia 1(2): 249. 1878.

TIPO: ITALIA. Sin localidad, en tallos arbolados y enraizados de Orchidaceae, s.f., Saccardo s.n. (tipo no localizado).

Hábito: saprobio, lignícola, crece sobre madera en descomposición.

Distribución: Ciudad de México (Medel et al., 2010); Nuevo León, Tamaulipas, Quintana Roo (San Martín y Rogers, 1989); Sonora (San Martín et al., 1999b); Veracruz (Medel et al., 1999); Hidalgo (Raymundo et al., 2016). Se cita por primera vez para Puebla.

Material estudiado: MÉXICO. Puebla, municipio Honey, riachuelo cerca del Río San Marcos, San Pedro Chila, 20¹7'38.78"'N, 98¹3'24.69"O, 17.IX.2017, M. Sánchez 942
(ENCB); Cascadas Arcoíris, Rincón de Chila, 20¹5'32.4"N, 98¹4'48.3"O, 25.XI.2017, M. Sánchez 1105 (ENCB).

Notas taxonómicas: se caracteriza por estromas simples o ramificados, cilíndricos con el ápice agudo y estéril, color negro; ascas 130-206 × 6.5-8 $\mu \mathrm{m}$, cilíndricas, poro apical amiloide; ascosporas 13-18 × 4.5-6 $\mu \mathrm{m}$, elipsoides, color marrón, con línea germinal recta casi a todo lo largo de la ascospora.

* Xylaria brachiata Sacc., Annls. Mycol. 4(1): 75. 1906.

TIPO: REPÚBLICA DEMOCRÁTICA DEL CONGO. Kisantu, sobre troncos, s.f., Saccardo s.n. (espécimen tipo no designado, ni se menciona en cual herbario se depositó).

Hábito: saprobio, lignícola, crece sobre madera.

Distribución: Tamaulipas, Veracruz (San Martín y Rogers, 1989; 1995). Se cita por primera vez para Puebla.

Material estudiado: MÉXICO. Puebla, municipio Honey, camino a la Cascada Velo de Novia, Chila de Juárez, 20¹7'22.41"N, 98¹3'69.67"O, 21.X.2017, M. Sánchez 1056 (ENCB).

Notas taxonómicas: se caracteriza por estromas simples o ramificados, cilíndricos a fusoides con el ápice estéril; ascas 130-160 × 7-8 $\mu \mathrm{m}$, cilíndricas, poro apical amiloide; ascosporas 11-14 × 5-6 $\mu \mathrm{m}$, elipsoides, color marrón, con línea germinal a lo largo de toda la ascospora.

\section{* Xylaria corniculata Sacc., Annls. Mycol. 4(1): 75. 1906.}

TIPO: REPÚBLICA DEMOCRÁTICA DEL CONGO. Kisantu, sobre troncos, s.f., Saccardo s.n. (espécimen tipo no designado, ni se menciona en cual herbario se depositó).

Hábito: saprobio, lignícola, crece sobre madera.

Distribución: Hidalgo (Raymundo et al., 2019); Guerrero (Medel et al., 2010); Tamaulipas (San Martín y Rogers, 1989); Veracruz (Medel et al., 2008). Se cita por primera vez para Puebla. 
Material estudiado: MÉXICO. Puebla, municipio Honey, riachuelo cerca del Río San Marcos, San Pedro Chila, 20¹7'38.78'N, 98¹3'24.69"O, 17.IX.2017, M. Sánchez 945 (ENCB); camino a la Cascada Velo de Novia, Chila de Juárez, 20¹7'22.41"N, 98¹3'69.67"O, 21.X.2017, M. Sánchez 1050-A (ENCB); Cascadas Arcoíris, Rincón de Chila, 20¹5'32.4"N, 98¹4'48.3"O, 25.XI.2017, M. Sánchez 1081 (ENCB); loc. cit., 26.II.2018, M. Sánchez 1152 (ENCB).

Notas taxonómicas: esta especie se caracteriza por estromas simples o ramificados, claviformes o irregulares, con ápice redondeado y estéril; ascas 144-186 × 7.5-9 $\mu \mathrm{m}$, cilíndricas, poro apical amiloide; ascosporas 16.5-20 × 6-7 $\mu \mathrm{m}$, elipsoides, color marrón, línea germinal espiralada.

*Xylaria curta Fr., Nova Acta R. Soc. Scient. Upsal., Ser. 3, 1(1): 126. 1851.

TIPO: ESTADOS UNIDOS DE AMÉRICA. Hawái, Isla Oahu, sobre troncos, s.f., Didrichs 28 (no se menciona en cual herbario se depositó el espécimen tipo).

इXylosphaera curta (Fr.) Dennis, Kew Bull. 13(1): 103. 1958.

Hábito: saprobio, lignícola, crece sobre madera.

Distribución: Campeche, Morelos (Medel et al., 2010), Tamaulipas (San Martín y Rogers, 1989); Veracruz (Welden y Lemke, 1961). Se cita por primera vez para Puebla.

Material estudiado: MÉXICO. Puebla, municipio Honey, camino a la Cascada Velo de Novia, Chila de Juárez, $20^{\circ} 17^{\prime} 22.41^{\prime \prime} \mathrm{N}, 98^{\circ} 13^{\prime} 69.67^{\prime \prime O}, 21 . X .2017$, M. Sánchez 1057 (ENCB).

Notas taxonómicas: se caracteriza por estromas simples o ramificados, cilíndricos a clavados con el ápice redondeado y fértil; ascas 117-158 × 5-6.5 $\mu \mathrm{m}$, cilíndricas, poro apical amiloide; ascosporas 9-11 × 4-4.5 $\mu \mathrm{m}$, elipsoides, color marrón, con línea germinal recta casi a lo largo de la ascospora.
*Xylaria enteroleuca (J.H. Mill.) P.M.D. Martin, South African Journal of Botany 36(2): 100. 1970.

इ Hypoxylon enteroleucum Speg. Fung. Argent. 264. 1898. TIPO. ARGENTINA. Santa Catarina, Buenos Aires, sobre madera de Gleditsia triacanthos, VIII.1890, Spegazzini s.n. (holotipo: LPS).

इ Penzigia enteroleuca J.H. Mill. Mycologia 32(2): 183. 1940.

Hábito: saprobio, lignícola, crece sobre madera.

Distribución: Chiapas, Jalisco, Nuevo Léon, Tamaulipas, Veracruz (San Martín y Rogers, 1995); Hidalgo (Raymundo et al., 2019). Se cita por primera vez para Puebla.

Material estudiado: MÉXICO. Puebla, municipio Honey, Cascadas Arcoíris, Rincón de Chila, 20¹5'32.4"N, 98¹4'48.3"O, 26.II.2018, T. Raymundo 7358 (ENCB); loc. cit., 26.II.2018, V. Rodríguez-León 23 (ENCB); loc cit., 19.XI.2016, M. Sánchez 831 (ENCB, FEZA); loc. cit., 26.II.2018, M. Sánchez 1155 (ENCB); loc. cit., 26.II.2018, R. Valenzuela 18028 (ENCB).

Notas taxonómicas: esta especie se caracteriza por ascas 90-95 × 7-8 $\mu \mathrm{m}$, cilíndricas, poro apical amiloide; ascosporas 8-12 × 6-7 $\mu \mathrm{m}$, color marrón, elipsoidales, con línea germinal recta. Se diferencia de $X$. berteroi por sus ascosporas de 9-14 × 6-8 $\mu \mathrm{m}$.

*Xylaria feejeensis (Berk.) Fr., Nova Acta R. Soc. Scient. Upsal. Ser. 3, 1(1): 128. 1851.

इ Sphaeria feejeensis (Berk.) Fr., Nova Acta R. Soc. Scient. Upsal., Ser.3, 1(1): 128. 1851. TIPO: ISLAS FIJI. Sin localidad, s.f., Berkeley s.n. (holotipo: K 34195).

三Xylosphaera feejeensis (Berk.) Dennis, Kew Bull. 13(1): 103. 1958

Hábito: saprobio, lignícola, crece sobre madera.

Distribución: Chiapas, Estado de México, Nuevo León, Tamaulipas (San Martín y Rogers, 1989); Sonora (San Martín et al., 1999b); Veracruz (Welden y Guzmán, 1978). Se cita por primera vez para Puebla. 
Material estudiado: MÉXICO. Puebla, municipio Honey, carretera Acahuales - Chila, $20^{\circ} 15^{\prime} 45.3^{\prime \prime} \mathrm{N}, 98^{\circ} 11^{\prime} 58.3^{\prime \prime O}$, 26.XI.2017, M. Sánchez 1118 (ENCB).

Notas taxonómicas: se caracteriza por estromas cilíndricos a clavados, espatulados, con el ápice redondeado y fértil; ascas $102-135 \times 4.5-5.5 \mu \mathrm{m}$, cilíndricas, poro apical amiloide; ascosporas 8.5-12 × 4.5-5 $\mu \mathrm{m}$, elipsoidales, color marrón, con línea germinal recta casi a lo largo de toda la ascospora.

*Xylaria juruensis Henn., Hedwigia 43(4): 262. 1904.

TIPO: BRASIL. Río Juruá, Juruá - Mirim, sobre palmera, VI.1901, Hennings 2860 (no se menciona en cual herbario se depositó el espécimen tipo).

इXylosphaera juruensis (Henn.) Dennis, Kew Bull. 13(1): 104. 1958.

Hábito: saprobio, lignícola, sobre madera en descomposición.

Distribución: Quintana Roo (San Martín y Rogers, 1989); Veracruz (Medel et al., 2008). Se cita por primera vez para Puebla.

Material estudiado: MÉXICO. Puebla, municipio Honey, Cascadas Arcoíris, Rincón de Chila, 20¹5'32.4"N, 98¹4'48.3"O, 26.II.2018, M. Sánchez 1144 (ENCB).

Notas taxonómicas: esta especie se caracteriza por estromas simples a ramificados, cilíndricos, con el ápice agudo y estéril; ascas 127-152 × 6-7 $\mu \mathrm{m}$, cilíndricas, poro apical amiloide; ascosporas 15-18 × 5-6 $\mu \mathrm{m}$, elipsoides, color marrón, línea germinal recta casi a lo largo de toda la ascospora.

*Xylaria laevis Lloyd, Mycol. Notes (Cincinnati) 65(5): 8. 1918.

TIPO: ESTADOS UNIDOS DE AMÉRICA. Cincinnati, s.f., Loyd s.n. (espécimen tipo no designado ni se menciona en cual herbario se depositó).
Hábito: saprobio, lignícola, crece sobre madera.

Distribución: Estado de México, Jalisco, Morelos, Querétaro (Medel et al., 2010); Hidalgo (Raymundo et al., 2016); Tamaulipas (San Martín y Rogers, 1989); Veracruz (Medel et al., 2008). Se cita por primera vez para Puebla.

Material estudiado: MÉXICO. Puebla, municipio Honey, Cascadas Arcoíris, Rincón de Chila, 20¹5'32.4"N, 98¹4'48.3'O, 25.XI.2017, M. Sánchez 1089 (ENCB).

Notas taxonómicas: esta especie se caracteriza por estromas de 16-23 mm de longitud, cilíndricos, superficie lisa, ápices redondeados y fértiles que al madurar se colapsan; ascas 105-195 × 5-6 $\mu \mathrm{m}$, cilíndricas, poro apical amiloide; ascosporas 9-12 × 4-5 $\mu \mathrm{m}$, elipsoidales, color marrón, línea germinal recta.

*Xylaria liquidambaris J.D. Rogers, Y.M. Ju \& F. San Martín (as "liquidambar"), Sydowia 54(1): 92. 2002.

TIPO: ESTADOS UNIDOS DE AMÉRICA. Florida, Alachua Country, sobre frutos de Liquidambar styraciflua, VII.1972, M. J. Berkeley y M. A. Curtis s.n. (holotipo: WSP 72081).

Hábito: endófito de frutos de Liquidambar styraciflua L.

Distribución: Hidalgo (Raymundo et al., 2016; 2019); Tamaulipas (San Martín y Rogers, 1989); Veracruz (Medel et al., 2008). Se cita por primera vez para Puebla.

Material estudiado: MÉXICO. Puebla, municipio Honey, camino a la Cascada Velo de Novia, Chila de Juárez, 20¹7'22.41"N, 98¹3'69.67"O, 21.X.2017, M. Sánchez 1055 (ENCB).

Notas taxonómicas: esta especie se caracteriza por crecer sobre frutos de Liquidambar styraciflua; ascas $170 \times$ 6-7 $\mu \mathrm{m}$, cilíndricas, con poro amiloide; ascosporas 13.5-16 $\times$ 5-6 $\mu \mathrm{m}$, elipsoides, color marrón, línea germinal recta.

Xylaria multiplex (Kunze) Fr., Nova Acta R. Soc. Scient. Upsal., Ser. 3, 1(1): 127. 1851. 
三 Sphaeria multiplex Kunze, Linnaea 5: 536. 1830. TIPO: SURINAME. Sin localidad, s.f., Kunze s.n. (tipo no localizado). इ Hypoxylon multiplex (Kunze) Mont., Annls. Sci. Nat., Bot. Sér. 2 13: 349. 1840.

इXylosphaera multiplex (Kunze) Dennis, Kew Bull. 13(1): 105. 1958.

Hábito: saprobio, lignícola, sobre madera en descomposición.

Distribución: Campeche, Nuevo León, Tabasco, Yucatán (Medel et al., 2010); Morelos, Nuevo León, Oaxaca (Pérez-Silva, 1975); Nuevo León, Quintana Roo, Veracruz (San Martín y Rogers, 1989). Puebla (Martínez-Alfaro et al., 1983); Veracruz (Medel et al., 2008).

Material estudiado: MÉXICO. Puebla, municipio Honey, Cascadas Arcoíris, Rincón de Chila, 20¹5'32.4"N, 98¹4'48.3"O, 25.XI.2017, M. Sánchez 1093 (ENCB).

Notas taxonómicas: se caracteriza por estromas simples o ramificados, cespitosos, con ápices estériles; ascas 109-142 × 5-6 $\mu \mathrm{m}$, cilíndricas, poro apical amiloide; ascosporas 9-12 × 4-4.5 $\mu \mathrm{m}$, elipsoidales, color marrón, línea germinal recta.

*Xylaria scruposa (Fr.) Fr. (as "scruposae"), Nova Acta R. Soc. Scient. Upsal., Ser. 3, 1(1): 127. 1851.

इ Sphaeria scruposa Fr., Elench. Fung. (Greifswald) 2: 55. 1828. TIPO: BRASIL. Sin localidad, sobre troncos, s.f., Fries s.n. (tipo no localizado).

इ Hypoxylon scruposum (Fr.) Mont., Annls. Sci. Nat. Bot., Sér. 2 13: 349. 1840.

三Xylosphaera scruposa (Fr.) Dennis, Kew Bull. 13(1): 106. 1958.

Hábito: saprobio, lignícola, crece sobre madera.

Distribución: Chiapas, Michoacán, Veracruz (San Martín y Rogers, 1989); Oaxaca (Raymundo et al., 2014); Veracruz (Medel et al., 2008). Se cita por primera vez para Puebla.
Material estudiado: MÉXICO. Puebla, municipio Honey, Parque Turístico El Sabinal, $20^{\circ} 14^{\prime} 05.1^{\prime \prime} \mathrm{N}, 98^{\circ} 12^{\prime} 49.8^{\prime \prime} \mathrm{O}$, 17.XI.2016, M. Sánchez 756 (ENCB, FEZA); Cascadas Arcoíris, Rincón de Chila, 20¹5'32.4"N, 98¹4'48.3"O, 25.XI.2017, M. Sánchez 1078 (ENCB), 1098 (ENCB); loc. cit., 26.II.2018, S. Rangel 1 (ENCB); loc. cit., 26.II.2018, T. Raymundo 7365 (ENCB); loc. cit., 26.II.2018, U. Rodríguez y D. Jiménez 102 (ENCB); loc. cit., 26.II.2018, M. Sánchez 1148 (ENCB); loc. cit., 26.II.2018, R. Valenzuela 18034 (ENCB).

Notas taxonómicas: se caracteriza por estromas simples a poco ramificados, cilíndricos, textura rugosa; ascas $188-224 \times 7.5-8 \mu \mathrm{m}$, cilíndricas, poro apical amiloide; ascosporas 18-21 × 6-7 $\mu \mathrm{m}$, elipsoidales, color marrón, con línea germinal oblicua o espiralada.

*Xylaria xylarioides (Speg.) Hladki \& A.I. Romero, Fungal Diversity 42: 86. 2010.

इ Hypoxylon xylarioides Speg. (as "xylaroide"), Anal. Soc. Cient. Argent. 9(4): 179.1880. TIPO: ARGENTINA. Río de la Plata, sobre tallos podridos de Jussiaea sp., III.1880, Spegazzini s.n. (holotipo: LPS 1972).

Hábito: saprobio, lignícola, sobre madera.

Distribución: Veracruz (Tapia et al., 2017). Se cita por primera vez para Puebla.

Material estudiado: MÉXICO. Puebla, municipio Honey, Cascadas Arcoíris, Rincón de Chila, 20¹5'32.4"N, 98¹4'48.3"O, 26.II.2018, M. Sánchez 1158 (ENCB).

Notas taxonómicas: esta especie se caracteriza por estromas cilíndricos, con ápice estéril, color negro, estipitado, tomentoso; ascas 129.2-144 × 7.6-9.6 $\mu \mathrm{m}$, cilíndricas, con poro apical amiloide; ascosporas (16)17.5-22.5 × 8-9 $\mu \mathrm{m}$, elipsoidales a naviculares, color marrón, línea germinal recta a casi todo lo largo de la espora.

\section{Discusión}

Las familias mejor representadas de Ascomycetes fueron Xylariaceae y Lachnaceae con 15 y cuatro especies respec- 
tivamente, seguidos de Hypoxylaceae, Nectriaceae, Orbiliaceae, Pyronemataceae y Sarcosomataceae, con tres especies cada una. El género más diverso es Xylaria con 14 especies. La especie más frecuente es Annulohypoxylon thouarsianum con 11 ejemplares.

En el bosque mesófilo de Honey se encontraron los cinco grupos funcionales de hongos definidos por PorrasAlfaro y Bayman (2011): endófitos (cuatro especies, 7.6\%), saprobios (36,70\%), epífitos (ocho, 15.3\%), micorrizógenos y patógenos (dos especies en cada uno, 3.8\% respectivamente). Guzmán (1995) mencionó que los ascomicetos son un grupo pobremente conocido en México, pero que dentro de los grupos con mayor número de taxones citados se encuentran los Xylariales, datos que coinciden con los trabajos de Medel (2013) para el listado de hongos de bosque mesófilo; en el grupo está representado con 42 especies, lo cual equivale a $40 \%$ del total. Sin embargo, menciona que los géneros mejor representados son Hypoxylon Bull. y Xylaria, lo que coincide con el presente trabajo.

Dentro de las especies encontradas en el presente estudio, los saprobios son los hongos más citados y mejor conocidos en el mundo, los ascomicetes epífitos son Cosmospora ustulinae (Nectriaceae) y Orbilia xanthostigma (Orbiliaceae), ambos crecen sobre otros hongos pertenecientes a la familia Xylariaceae. Helvella solitaria y Leotia lubrica son los únicos taxones en el estudio que son micorrizógenos y están asociados a las raíces de Quercus. Finalmente, las especies patógenas fueron Biscogniauxia nummularia y Kretzschmaria deusta, ambas atacan a Quercus sp.

El conocimiento de los ascomicetos del bosque mesófilo del país se ha incrementado, sí consideramos que para este tipo de vegetación, Chacón y Medel (1993) realizaron la recopilación de Ascomicetes del mesófilo en México y citaron 96 especies; posteriormente Medel (2013) reportó 107 especies y Raymundo et al. (2016) describieron 14 nuevos registros de hongos para este tipo de vegetación. Recientemente, Raymundo et al. (2019) registraron 13 taxones para el bosque relicto de Fagus L. dentro del bosque mesófilo de la Sierra Alta de Hidalgo. Con la presente aportación se conocen 141 especies de ascomicetos para el bosque mesófilo de México de las cuales Annulohypoxylon fuscum, Bisporella citrina, B. sulphurina, Bulgaria inquinans, Hyalorbilia inflatula, Melogramma campylospora, Orbilia sarraziniana y Xylaria corniculata son consideradas características de este ecosistema según Medel (2013). De esta forma Adelphella babingtonii, Cookeina venezuelae, Cudoniella acicularis, Hymenoscyphus herrerae, Lachnum apalum, Scutellinia pennsylvanica y Xylaria xylarioides se incorporan a este listado de taxones exclusivos para el bosque mesófilo de montaña.

\section{Conclusiones}

Los ascomicetos son el grupo de hongos mejor representados en los bosques mesófilos de Puebla. Con el presente trabajo se incrementó el número de registros para el estado de Puebla con un total de 94 especies y para los bosques de niebla la cifra asciende a 141 para México. Se considera importante dar continuidad a los estudios ante la pérdida significativa e inevitable de áreas forestales. Es necesario incrementar los esfuerzos por inventariar y describir la riqueza fúngica y de otros organismos de este ecosistema.

\section{Contribución de autores}

MSF, MAHM y TR concibieron y diseñaron el estudio. MSF, $T R$ y RV realizaron las recolecciones y determinaciones de las especies. MSF, TR, RV, JGJ, MAHM y MMP contribuyeron a la adquisición de datos importantes para el trabajo. Las fotografías fueron tomadas por TR, RV y MSF. MSF y TR escribieron el manuscrito con ayuda de JGJ. Todos los autores contribuyeron a la discusión, revisión y aprobación del manuscrito final.

\section{Financiamiento}

Este estudio fue apoyado por el Consejo Nacional de Ciencia y Tecnología con el proyecto 252935 y por el Instituto Politécnico Nacional (IPN), a través de la Secretaria de Investigación y Posgrado (SIP) en el proyecto 20200248, 20200956.

\section{Agradecimientos}

MAHM y MSF agradecen a Alejandrina Ávila Ortiz por el apoyo brindado para la realización al inicio de este estudio, así como a las autoridades de FES Zaragoza. RV agradece, además, a la Comisión de Operación y Fomento de las Actividades Académicas (COFAA) del IPN y a la Secretaria de 
Posgrado e Investigación de la misma institución por el apoyo recibido mediante las becas de exclusividad (SIBE y EDI) para realizar sus investigaciones.

\section{Literatura citada}

Alexopoulos, C. J. y C. W. Mins. 1985. Introducción a la micología. Ediciones Omega. Barcelona, España. 638 pp.

Álvarez, V. I., T. Raymundo y R. Valenzuela. 2016. Hongos Histerioides (Dothideomycetes, Ascomycota) del bosque tropical caducifolio en el Parque Nacional Lagunas de Chacahua, Oaxaca, México. Acta Botanica Mexicana 116: 49-64. DOI: https://doi.org/10.21829/abm116.2016.1118

Barbosa-Reséndiz, A., R. Valenzuela, M. Sánchez-Flores, S. Bautista-Hernández, A. Cobos-Villagrán, A. Pérez-Valdespino, R. Espinoza-Mellado, M. Martínez-Pineda y T. Raymundo. 2020. El género Daldinia (Sordariomycetes, Ascomycota) en México. Acta Botanica Mexicana 127: e1600. DOI: https:// doi.org/10.21829/abm127.2020.1600

Bautista, N., S. Chacón y G. Guzmán. 1986. Ascomicetes poco conocidos de México. III. Especies del Estado de Morelos. Revista Mexicana de Micología 2: 85-104.

Berkeley, M. J. y C. E. Broome. 1851. XVI Notices of Brithish fungi. The Annals and Magazine of Natural History, Serie 2(7): 176-189.

Beug, M., A. E. Bessette y A. R. Bessette. 2014. Ascomycete Fungi of North America a Mushroom Reference Guide. University of Texas Press. Texas, USA. 488 pp. DOI: https://doi. org/10.7560/754522

Boudier, M. 1888. Nouvelles espèces de Discomycetes inoperculés de France. Bulletin de la Société Mycologique de France 4(2):76-86.

Brayford, D., B. M. Honda, F. R. Mantiri y G. J. Samuels. 2004. Neonectria and Cylindrocarpon: the Nectria mammoidea group and species lacking microconidia. Mycologia 96(3): 572-597. DOI: https://doi.org/10.1080/15572536.2005.11832955

Breitenbach, J. y F. Kränzlin. 1984. Fungi of Switzerland, Vol. I. Ascomycetes. Mykologia. Lucerna, Switzerland. 313 pp.

Bulliard, J. B. F. 1790. Champignons de la France. Herbier de la France 10: 473.

Calonge, F. D., M. Mata y L. Umaña. 2006. The genus Phillipsia (Ascomycota) in Costa Rica; with a key to identify the species. Boletín de la Sociedad Micológica de Madrid 30: 35-42.

Castillo, J., J. García y F. San Martín. 1979. Algunos datos sobre la distribución ecológica de los hongos, principalmente los micorrícicos, en el centro del Estado de Nuevo León. Boletín de la Sociedad Mexicana de Micología 13: 229-237.

Chacón, S. y G. Guzmán. 1983. Ascomycetes poco conocidos de México. Boletín de la Sociedad Mexicana de Micología 18: 183-218. Chacón, S. y R. Medel. 1993. Los hongos (principalmente macromicetos) registrados en el bosque mesófilo de montaña de México. In: Marmolejo, J. y F. Garza-Ocañas (eds.). Contribuciones micológicas en homenaje al Biól. José Castillo Tovar, por su labor en pro de la micología mexicana. Número especial 13. Facultad de Ciencias Forestales, Universidad Autónoma de Nuevo León, Linares, México. Pp. 61-110.

Chacón, S. y F. Tapia-Padilla. 2013. Algunas especies del género Byssosphaeria (Melanommataceae, Pleosporales) de Veracruz, México. Revista Mexicana de Biodiversidad 84(3): 739745. DOI: https://doi.org/10.7550/rmb.32985

Chacón, S., F. Tapia y M. Esqueda. 2014. New records of Dothideomycetes from Mexico. Mycotaxon 128: 147-157. DOI: https://doi.org/10.5248/128.145

Chavarria, A., M. C. González, E. Dantán y J. Cifuentes. 2010. Evaluación espacial y temporal de la diversidad de los ascomicetes dulceacuícolas del canal turístico Santa Cruz, Xochimilco, México. Revista Mexicana de Biodiversidad 81: 733-744.

Chaverri, P., S. Huhndorf, J. D. Rogers y G. J. Samuels. 2011. Microhongos comunes de Costa Rica y otras regiones tropicales. Instituto Nacional de Biodiversidad. Santo Domingo de Heredia, Costa Rica. 241 p.p.

Chio, R. E., I. Frutis y G. Guzmán. 1988. Hongos del Estado de México. I. Especies citadas de la bibliografía, primera parte. Ascomycetes, Tremellales y Aphyllophorales. Revista Mexicana de Micología 4: 97-113.

Cifuentes, J., M. Villegas y L. Pérez-Ramírez. 1986. Hongos. In: Lot, A. y F. Chiang (eds.). Manual de Herbario. Consejo Nacional de la Flora de México, A.C. México, D.F., México. Pp. 55-64.

Cifuentes, J., M. Villegas, L. Pérez-Ramíirez, M. Bulnes, V. Corona, M. R. González, I. Jiménez, A. Pompa y G. Vargas. 1990. Observaciones sobre la distribución, hábitat e importancia de los hongos de Los Azufres, Michoacán. Revista Mexicana de Micología 6: 133-149.

Denison, W. C. 1969. Central American Pezizales III. The genus Phillipsia. Mycologia 61: 289-304. DOI: https://doi. org/10.2307/3757123

Dennis, R. W. G. 1981. British Ascomycetes. Ed. J. Cramer. Vaduz, Germany. 1959 pp. 
Díaz-Barriga, H., F. Guevara y R. Valenzuela. 1988. Contribución al conocimiento de los macromicetos del estado de Michoacán. Acta Botanica Mexicana 2: 21-44. DOI: https://doi. org/10.21829/abm2.1988.564

Frutis, I. y G. Guzmán. 1983. Contribución al conocimiento de los hongos del estado de Hidalgo. Boletín de la Sociedad Mexicana de Micología 18: 219-266.

Galán, R., A. Raitviir, N. Ayala y C. Ochoa. 1994. First contribution to the knowledge of the Leotiales of Baja California and adjacent areas. Mycological Research 98(10): 1137-1152. DOI: https://doi.org/10.1016/S0953-7562(09)80199-8

Gándara, E., L. Guzmán-Dávalos, G. Guzmán y O. Rodríguez. 2014. Inventario micobiótico de la región de Tapalpa, Jalisco, México. Acta Botanica Mexicana 107: 165-185.DOI: https:// doi.org/10.21829/abm107.2014.207

Garza, F., J. García y J. Castillo. 1985. Macromicetos asociados al bosque de Quercus rysophylla en algunas localidades del centro del Estado de Nuevo León. Revista Mexicana de Micología 1: 423-437.

Guzmán, G. 1995. La Diversidad de Hongos en México. Ciencia 39: 52-57.

Hansen, K., D. H. Pfister y D. S. Hibbet. 1999. Phylogenetic relationships among species of Phillipsia inferred from molecular and morphological data. Mycologia 91(2): 299-314. DOI: https://doi.org/10.1080/00275514.1999.12061020

Hengstmengel, J. 1996. Notes on Hymenoscyphus. 2. On 3 non-fructicolous species of the fructigenus-group with croziers. Persoonia 16(2): 191-207.

Heredia, G. 1989. Estudio de los hongos de la Reserva de la Biosfera El Cielo, Tamaulipas. Consideraciones sobre la distribución y ecología de algunas especies. Acta Botanica Mexicana 7: 1-18. DOI: https://doi.org/10.21829/abm7.1989.577

Herrera, M. J., L. Guzmán-Dávalos y O. Rodríguez. 2002. Contribución al conocimiento de la micobiota de la región de San Sebastián del Oeste, Jalisco, México. Acta Botanica Mexicana 58: 19-50. DOI: https://doi.org/10.21829/abm58.2002.888

Index Fungorum. 2020. Index fungorum base de datos. http://www. indexfungorum.org/Names/Names.asp (consultado febrero de 2020).

INEGI. 2009. Prontuario de información geográfica municipal de los Estados Unidos Mexicanos. Instituto Nacional de Estadística y Geografía Honey, Puebla. México. 9 pp. lqbal, S. H. 1972. Gaeumannomyces leptosporus, a new aquatic Ascomycete. Transactions British Mycological Society 58(2): 346-348.

Ju, Y. M., J. D. Rogers, F. San Martín y A. Granmo. 1998. The genus Biscogniauxia. Mycotaxon 66: 1-98.

Karsten, P. A.1869. Fungi quídam novi fennici. Notiser ur Sällskapets pro Fauna et Flora Fennica Förhandlingar 10: 327-330.

Kirk, P. M., P. F. Cannon, D. W. Minter y J. A. Stalpers. 2008. Ainsworth and Bisby's Dictionary of the fungi. $10^{\text {th }}$ ed. CAB International. Wallingford, UK. 771p.

Kornerup, A. y J. H. Wanscher. 1978. Methuen Handbook of Colour. 3a. ed. Eyre Methuen, London, UK. 252 pp.

Kučera, V. 2011. Cudoniella acicularis. Catathelasma 13: 25-27. Landeros, F. y L. Guzmán-Dávalos. 2013. Revisión del género Helvella (Ascomycota: Fungi) en México. Revista Mexicana de Biodiversidad 84: S3-S20. DOI: https://doi.org/10.7550/rmb.31608

López, A. y J. García. 2002. Stictis radiata, Ascomycota: Ostropales: Stictidaceae. Funga Veracruzana 69: 1-2.

López, A. y J. García.2010. Hyalorbilia inflatula. Funga Veracruzana 106: 1-4

Martínez-Alfaro, M. A., E. Pérez-Silva y E. Aguirre-Acosta. 1983. Etnomicología y exploraciones micológicas de la Sierra Norte de Puebla. Boletín de la Sociedad Mexicana de Micología 18: 51-64. DOI: https://doi.org/10.33885/sf.1983.2.574

Medel, R. 2013. Hongos ascomicetos del bosque mesófilo de montaña en México. Acta Botanica Mexicana 105: 87-106. DOI: https://doi.org/10.21829/abm105.2013.224

Medel, R. y S. Chacón. 2000. Contribución al conocimiento del género Plectania (Pezizales, Sarcosomataceae) en México. Acta Botanica Mexicana 50: 11-19. DOI: https://doi. org/10.21829/abm50.2000.839

Medel, R., F. D. Calonge y G. Guzmán. 2006. Nuevos registros de Pezizales (Ascomycota) de Veracruz. Revista Mexicana de Micología 23: 83-86

Medel, R., R. Castillo y G. Guzmán. 2008. Las especies de Xylaria (Ascomycota, Xylariaceae) conocidas de Veracruz, México y discusión de nuevos registros. Revista Mexicana de Micología 28: 101-118.

Medel, R., G. Guzmán y R. Castillo. 2010. Adiciones al conocimiento de Xylaria (Ascomycota, Xylariales) en México. Revista Mexicana de Micología 31: 9-18.

Medel, R., G. Guzmán y S. Chacón. 1999. Especies de macromicetos citadas de México IX. Ascomycetes, parte III: 1983- 
1996. Acta Botanica Mexicana 46: 57-72. DOI: https://doi. org/10.21829/abm46.1999.816

Méndez-Mayboca, F. C., S. Chacón, M. L. Coronado y M. Esqueda. 2007. Ascomycetes de Sonora, México, II: Reserva Forestal Nacional y Refugio de Fauna Silvestre Ajos-Bavispe. Revista Mexicana de Micología 25: 33-40.

Ortega-López, I., R. Valenzuela, A. D. Gay-González, M. B. N. Lara-Chávez, E. O. López-Villegas y T. Raymundo. 2019. La Familia Sarcoscyphaceae (Pezizales, Ascomycota) en México. Acta Botanica Mexicana 126: e1430. DOI: https://doi. org/10.21829/abm126.2019.1430

Paden, J. W. 1983. Sarcosomataceae (Pezizales, Sarcoscyphineae). Flora Neotropica Monographs 37: 1-17.

Pérez-Silva, E. 1975. El género Xylaria (Pyrenomycetes) en México, I. Boletín de la Sociedad Mexicana de Micología 9: 31-52.

Pérez-Silva, E., M. Esqueda-Valle y A. Armenta-Calderón. 1996. Ascomycetes de Sonora I: Discomycetes y Pyrenomycetes. Revista Mexicana de Micología 12: 97-106.

Persoon, C. H. 1801. Peziza. Synopsis Methodica Fungorum 2: 433-480.

Pfister, D. H., N. Matočec y I. Kušan. 2008. Integrated studies in the classification of the Pezizaceae. Re-evaluation of the genus Pachyella with a new seggregate genus Adelphella. Mycologia Montenegrina 11: 7-17.

Pompa-González, A. y J. Cifuentes. 1991. Estudio taxonómico de los Pezizales de los estados de Guerrero, Hidalgo, Estado de México y Michoacán. Revista Mexicana de Micología 7: 87-112.

Porras-Alfaro, A. y P. Bayman. 2011. Hidden Fungi, Emergent Properties: endophytes and microbiomes. Annual Review of Phytopathology 49: 291-315. DOI: https://doi.org/10.1146/ annurev-phyto-080508-081831

Ramírez-López, I. y M. Villegas-Ríos. 2007. El conocimiento taxonómico de Geoglossaceae sensu lato (Fungi: Ascomycetes) en México con énfasis en la zona centro y sur. Revista Mexicana de Micología 25: 41-49.

Raymundo, T., R. Díaz-Moreno, S. Bautista-Hernández, E. Aguirre-Acosta y R. Valenzuela. 2012. Diversidad de ascomicetes macroscópicos en Bosque Las Bayas, municipio de Pueblo Nuevo, Durango, México. Revista Mexicana de Biodiversidad 83: 1-14. DOI: https://doi.org/10.22201/ ib.20078706e.2012.1.1241

Raymundo, T., M. Martínez-Pineda, A. Cobos-Villagrán, M. Sánchez-Flores y R. Valenzuela. 2020. Primer registro de Ungui- culariopsis ravenelii (Leotiomycetes, Ascomycota) en México. Acta Botanica Mexicana 127: e1666. DOI: https://doi. org/10.10.2189/abm127.2020.1666

Raymundo, T., R. Soto-Agudelo, S. Bautista-Hernández, A. Morales-Campos y R. Valenzuela. 2016. Catálogo de los ascomicetos del bosque mesófilo de montaña de Tlanchinol, Hidalgo (México). Boletín de la Sociedad Micológica de Madrid 40: 79-104. DOI: https://doi.org/10.21829/abm120.2017.1263 Raymundo, T., E. Escudero-Leyva, I. Ortega-López, D. Castro-Bustos, H. León-Avedaño y R. Valenzuela. 2014. Ascomicetos del bosque tropical caducifolio en el Parque Nacional Lagunas de Chacahua, Oaxaca, México. Boletín de la Sociedad Micológica de Madrid 38: 9-21.

Raymundo, T., E. Escudero-Leyva, R. Soto-Agudelo, J. García-Jiménez, L. Romero-Bautista y R. Valenzuela. 2017. Nuevos registros de Hypocreales (Sordariomycetes, Ascomycota) del bosque mesófilo de montaña de la Sierra Alta Hidalguense en México. Acta Botanica Mexicana 120: 39-57. DOI: https://doi.org/10.21829/abm120.2017.1263

Raymundo, T., E. Aguirre-Acosta, S. Bautista-Hernández, M. Contreras-Pacheco, P. Garma, H. León-Avedaño y R. Valenzuela. 2013. Catálogo de los Ascomycota en los bosques de Santa Martha Latuvi, Sierra Norte, Oaxaca, México. Boletín de la Sociedad Micológica de Madrid 37: 13-29.

Raymundo, T., R. Valenzuela, Y. García-Martínez, M. A. BravoÁlvarez, J. C. Ramírez- Martínez, S. Bautista-Hernández, M. Palacios-Pacheco y I. Luna-Vega. 2019. Ascomycetes (Fungi) from the relic forest of Fagus grandifolia subsp. mexicana in Eastern Mexico. Phytotaxa 418(1): 001-041. DOI: https:// doi.org/10.11646/phytotaxa.418.1.1

Requejo, O. y M. L. Castro. 2015. Mycobiota de las Gándaras de Budiño (Pontevedra, N. O. Peninsula Ibérica), Ascomycota. Boletín de la Sociedad Micológica de Madrid 39: 75-082

Reyes-Díaz, M. 2003. Estudio de la vegetación de los municipios de Honey y Pahuatlán de la Sierra Norte de Puebla, Puebla. Tesis de licenciatura. Facultad de Ciencias, Universidad Nacional Autónoma de México. Cd. Mx., México. 81 pp.

Rodríguez-Alcántar, O., D. Figueroa-García y M. J. Herrera-Fonseca. 2018. Catálogo de los hongos del Volcán de Tequila, municipio de Tequila, Jalisco, México. Polibotánica 45: 15-33. DOI: https://doi.org/10.18387/polibotanica.45.3

Rogers, J. D. y Y.-M. Ju. 1998. The genus Kretzschmaria. Mycotaxon 68: 345-393. 
San Martín, F. y P. Lavín. 1997. Los Ascomycetes Acantonitschkia, Corynelia, Lopadostoma y Camarops en México. Acta Botanica Mexicana 41: 31-41. DOI: https://doi.org/10.21829/ abm41.1997.790

San Martín, F. y J. D. Rogers. 1989. A preliminary of account of Xylaria of Mexico. Mycotaxon 34: 283-374.

San Martín, F. y J. D. Rogers. 1993. Biscogniauxia and Camillea in Mexico. Mycotaxon 47: 229-258.

San Martín, F. y J. D. Rogers. 1995. Notas sobre la historia y relaciones de hospedaje y distribución del género Xylaria (Pyrenomycetes, Sphaeriales) en México. Acta Botanica Mexicana 30: 21-40. DOI: https://doi.org/10.21829/abm30.1995.731

San Martín, F. y J. D. Rogers. 1996. A preliminary list of xylariaceous fungi at El Cielo Biosphere reserve in Tamaulipas, México. Acta Botanica Mexicana 37: 23-31. DOI: https:// doi.org/10.21829/abm37.1996.766

San Martín, F., Y. M., Ju y J. D. Rogers. 1999a. Algunas especies de Hypoxylon (Pyrenomycetes, Xylariaceae) de México. Acta Botanica Mexicana. 47: 31-53. DOI: https://doi. org/10.21829/abm47.1999.823

San Martín, F., J. D. Rogers, P. Lavín, E. Pérez-Silva y M. Esqueda. 1999b. New Records of Xylariaceae of Sonora, Mexico. Mycotaxon 71: 129-134.

Schoch, C. L., G. H. Sung, F. López-Giráldez, J. P. Townsend, J. Miadlikowska, V. Hofstetter, B. Robbertse, B. Matheny, F. Kauff, Z. Wang, C. Gueidan, R. M. Andrie, K. Trippe, L. M. Ciufetti, A. Wynns, E. Fraker, B. P. Hodkinson, G. Bonito, Z. J. Groenewald, M. Arzanlou, G. Sybren de Hoog, P. W. Crous, D. Hewitt, D. Pfister, K. Peterson, M. Gryzenhout, M. Wingfield, A. Aptroot, S. Suh, M. Blackwell, D. M. Hillis, G. W. Griffith, L. A. Castlebury, A. Y. Rossman, A. Thorsten Lumbsch, R. Lüking, B. Büdel, A. Rauhut, P. Diederich, D. Ertz, D. M. Geiser, K. Hosaka, P. Inderbitzin, J. Kohlmeyer, B. Volkmann-Kohlmeyer, L. Mostert, K. O’Donnell, H. Sipman, J. D. Rogers, R. A. Shoemaker, J. Sugiyama, R. C. Summerbell, W. Untereiner, P. R. Johnston, S. Stenroos, A. Zuccaro, P. S. Dyer, P. Crittenden, M. S. Cole, K. Hansen, J. M. Trappe, R. Yahr, F. Lutzoni y J. W. Spatafora. 2009. The Ascomycota Tree of Life: a phylum-wide phylogeny clarifies the origin and evolution of fundamental reproductive and ecological traits. System- atic Biology 58: 224-239. DOI: https://doi.org/10.1093/sysbio/syp020

Shearer, C. A., J. L. Crane y W. Chen. 1999. Freshwater Ascomycetes: Ophioceras Species. Mycologia 91: 145-156. DOI: https://doi.org/10.1080/00275514.1999.12061004

Sierra, S., L. Izquierdo-San Agustín, S. Castro-Santiuste, I. Rodríguez-Gutiérrez, A. Alcántara-Mojica, L. Pérez-Ramírez y J. Cifuentes. 2016. Nuevos registros del género Scutellinia (Pyronemataceae, Pezizales) para la micobiota mexicana. Acta Botanica Mexicana 117: 79-89. DOI: https://doi. org/10.21829/abm117.2016.1169

Tapia, F., Y. M. Ju, S. Chacón y E. Utrera-Barillas. 2017. Five penzingioid Xylaria species from Veracruz (México). Mycotaxon 132: 19-27. DOI: https://doi.org/10.5248/132.19

Thind, K. S. y M. P. Sharma. 1980. The family Orbiliaceae in India (Helotiales). Sydowia Annals Mycologici Serie II 33: 300-310.

Ulloa, M. y R. T. Hanlin. 2006. Nuevo diccionario ilustrado de Micología. APS Press. St. Paul, USA. 615 pp.

Uzun, Y., I. Acar, M. E. Akçay e I. Akata. 2014. Additions to the Turkish Discomycetes. Turkish Journal of Botany 38: 617622.

Valenzuela, R. 1990. El género Chlorociboria en México. Revista Mexicana de Micología 6: 125-131.

Varela, L. y J. Cifuentes. 1979. Distribución de algunos macromicetos en el Noreste del Estado de Hidalgo. Boletín de la Sociedad Mexicana de Micología 13: 75-88.

Vite-Garín, T. M., J, L. Villarruel-Ordaz y J. Cifuentes. 2006. Contribución al conocimiento del género Helvella (Ascomycota: Pezizales) en México: Descripción de especies poco conocidas. Revista Mexicana de Biodiversidad 77: 143.151. DOI: https://doi.org/10.22201/ib.20078706e.2006.002.335

Welden, A. L. y G. Guzmán. 1978. Lista preliminar de los hongos, líquenes y mixomicetos de las regiones de Uxpanapa y Xalapa (parte de los estados de Veracruz y Oaxaca). Boletín de la Sociedad Mexicana de Micología 12: 59-102.

Welden, A. L. y P. Lemke. 1961. Notas sobre algunos hongos mexicanos. Boletín de la Sociedad Mexicana de Micología 26: 1-24. DOI: https://doi.org/10.17129/botsci.1067

Zhuang, W. Y. 1988. A monograph of the genus Unguiculariopsis (Leotiaceae, Encoelioide). Mycotaxon 32: 1-83. 